\title{
The Drosophila BEACH Family Protein, Blue Cheese, Links Lysosomal Axon Transport with Motor Neuron Degeneration
}

\author{
Angeline Lim ${ }^{1}$ and Rachel Kraut ${ }^{2}$ \\ ${ }^{1}$ Department of Molecular, Cell, and Developmental Biology, University of California, Santa Cruz, Santa Cruz, California 95064, and ${ }^{2}$ Institute of \\ Bioengineering and Nanotechnology, Agency for Science, Technology, and Research, Singapore 138669
}

\begin{abstract}
Impaired axon transport is one of the earliest pathological manifestations of several neurodegenerative diseases, and mutations in motor proteins can exacerbate or cause degeneration (Williamson and Cleveland, 1999; Gunawardena and Goldstein, 2004; Stokin and Goldstein, 2006). Compromised function in lysosomes and other degradative organelles that intersect with the lysosomal pathway are also strongly implicated in neurodegenerative disease pathology (Nixon and Cataldo, 2006; Rubinsztein, 2006). However, any functional link between these two phenomena has not as yet been recognized. Drosophila mutants in blue cheese (bchs) undergo progressive brain degeneration as adults and have shortened life span (Finley et al., 2003), but the cellular function of Bchs and the cause of degeneration have not been identified. A role in lysosomal trafficking is suggested by the homology of Bchs with the vesicle trafficking-associated BEACH (Beige and Chediak-Higashi) domain protein family (Wang et al., 2002; De Lozanne, 2003) and by its genetic interaction with a lysosomal transport pathway (Simonsen et al., 2007). Here, we describe the degeneration of a population of identified larval motor neurons in $b c h s$ mutants. We present evidence that Bchs is primarily lysosomal in those motor neurons in wild type and, using live fluorescence imaging of individual motor neurons in intact larvae, show that lysosomal vesicles fail to be transported toward motor neuron termini in $b c h s$ mutant and Bchs-overexpressing larvae. We suggest therefore that anterograde transport of lysosomes toward synaptic termini is a key factor in preventing motor neuron degeneration and that Bchs reveals a functional link between the lysosomal degradative pathway and transport.
\end{abstract}

Key words: degeneration; lysosome; transport; motoneuron; motor neuron; Drosophila; neuron death

\section{Introduction}

Failure in axon transport has been identified as an early pathogenic event in Alzheimer's disease and other neurodegenerative diseases, notably amyotrophic lateral sclerosis (ALS) and the polyglutamine diseases (Williamson and Cleveland, 1999; Gunawardena et al., 2003; Szebenyi et al., 2003; Chevalier-Larsen and Holzbaur, 2006; Stokin and Goldstein, 2006). The observations that kinesin mutations interact genetically with transgenic Alzheimer's disease models (Stokin et al., 2005) and that mutations in motor proteins lead to degenerative phenotypes (LaM-

\footnotetext{
Received June 6, 2008; revised Nov. 12, 2008; accepted Dec. 7, 2008.

This work was supported by funding from National Institutes of Health (NIH) R01 Research Grant NS28182 (to Kai Zinn) and NIH-National Institute of Neurological Disorders and Stroke National Research Service Award Grant 5F32NS010994-03 (R.K.), and funding to the Institute of Bioengineering and Nanotechnology by the Biomedical Research Council, Agency for Science, Technology, and Research (Singapore). We thank Miki Fujioka for evenskipped driver lines; Henry Chang, Markus Gonzalez-Gaitan, and Helmut Kraemer for the UAS-rab11-GFP, rab-5 and $2 \times$ FYVE-GFP, and LAMP-GFP stocks, respectively; and the LiQun Luo Laboratory for CD8-GFP lines. Kim Finley, Rita Khodosh, and Paul Garrity generously donated $b c h s$ mutant lines and shared unpublished data. We thank Ashley Wright for generating recombinant $b c h s$ marker lines and for discussions, and Kai Zinn for support. We are very grateful to Bill Saxton for critical reading of this manuscript, and to Alice Schmid, Anuradha Ratnaparkhi, Kim Finley, and Sarita Hebbar for helpful discussions.

Correspondence should be addressed to Rachel Kraut, School of Biological Sciences, Nanyang Technological University, 60 Nanyang Drive, Singapore 639798. E-mail: rskraut@ntu.edu.sg.

D0I:10.1523/JNEUROSCI.2582-08.2009
}

Copyright $\odot 2009$ Society for Neuroscience $\quad 0270-6474 / 09 / 290951-13 \$ 15.00 / 0$ onte et al., 2002; Hafezparast et al., 2003; Puls et al., 2003) have led to the proposal that transport defects are a primary cause of the neurodegenerative pathology. One possible explanation for the catastrophic effect of transport disruptions to neuronal survival was that axonal blockages would impede transport of growth factors to the cell body (Gunawardena and Goldstein, 2004; Salehi et al., 2006; Stokin and Goldstein, 2006); however, other mechanisms have been proposed (Ravikumar et al., 2005; Chevalier-Larsen and Holzbaur, 2006).

Although proper functioning of the axon transport machinery appears to be crucial to neuronal survival, a different line of investigation is based on the hypothesis that lysosomes and autophagosomes are important in preventing the accumulation of damaging aggregated proteins (Nixon, 2006; Rubinsztein, 2006). Disease progression is accompanied by a failure of degradative functions (Nixon et al., 2001; Bahr and Bendiske, 2002), and other evidence directly links the activities of some disease-related proteins to degradation (Cuervo et al., 2004; Small and Gandy, 2006). Compromised degradative function generally exacerbates or can lead to degeneration, whereas normal or excess activity can forestall the effects of neurodegeneration-causing conditions (Hara et al., 2006; Komatsu et al., 2006; Rubinsztein, 2006). So far, the functional link between these two disease-associated pathologies (axonal transport vs degradation) is not entirely clear. 
Mutations in Drosophila blue cheese (bchs) are associated with shortened life span and generalized brain degeneration (Finley et al., 2003), as well as interactions with the recycling endosomal protein rab11 (Khodosh et al., 2006). Bchs is a member of the Beige and Chediak-Higashi (BEACH) family of proteins whose role in vesicular trafficking has been described previously (Wang et al., 2000; Ward et al., 2000; Cornillon et al., 2002; Su et al., 2004; de Souza et al., 2007). The abnormal accumulation of ubiquitinated proteins in bchs brain tissue and the ability of lysosomal mutations to suppress a Bchs overexpression eye phenotype, suggested a possible role in degradation (Finley et al., 2003). However, Khodosh et al. (2006) found no association of Bchs protein with degradative (lysosomal) compartments. Therefore, the cellular function of the Bchs protein relating it to neurodegeneration is currently unclear. Moreover, its expression pattern in the CNS has not been correlated with any particular neuronal cell type that is affected in the mutant.

In this study, we present quantitative evidence that Bchs is present on endolysosomes and that it specifically affects anterograde transport of these vesicles in motor neurons at which Bchs is normally expressed. In contrast, loss of Bchs does not affect the mobility or direction of mitochondrial transport in the same motor neurons. In addition to axon transport defects in endolysosomal compartments, motor neurons of bchs mutant larvae display features typically associated with degeneration. Abnormal neuronal death in the CNS is shown by increased terminal deoxynucleotidyl transferase-mediated dUTP nick end labeling (TUNEL) reactivity and loss of even-skipped in affected neurons. Morphological changes similar to those described in other degenerative situations are also seen, including axonal varicosities, fragmentation, and disorganization of microtubules in axons and termini (Watts et al., 2003; Jablonka et al., 2004; Luo and O'Leary, 2005; Misgeld, 2005).

Given the recently reported genetic interactions between $b c h s$ and lysosomal transport proteins (Simonsen et al., 2007), and the data presented here, we suggest that Bchs promotes the transport of an endolysosomal compartment from the cell body to nerve termini and that a breakdown in this phenomenon contributes to the neurodegenerative phenotype.

\section{Materials and Methods}

Antibodies, immunofluorescence. Rabbit polyclonal antiserum (Covance Research Products) was generated against the 1008 C-terminal amino acids of the Bchs protein, by subcloning a Klenow-filled in XbaI-XhoI fragment of bchs cDNA LP02084 in pOT2 into SmaI-digested protein expression vector pGEX-3X (Glutagene; Amrad Corporation). Bchs GST (glutathione $S$-transferase) fusion protein was made as in the study by Kraut and Campos-Ortega (1996). Specificity of the antibody was tested by staining $b c h s$-deficient embryo fillets (supplemental Fig. S1, available at www.jneurosci.org as supplemental material) of the genotype $b c h s^{1}$ / $D f(2 L) c 17$, and by Western blotting to extracts of three third-instar larval brains per lane (supplemental Fig. S1, available at www.jneurosci.org as supplemental material). Anti-Bchs was used at 1:1000 for tissues and 1:6000 for blots.

Rabbit and mouse polyclonal antibodies to green fluorescent protein (GFP) (Living Colors, Biomed Diagnostics, and Invitrogen, respectively) were used at 1:1000. Alexa-coupled secondary antibodies (Invitrogen) were used at 1:1000. Mouse Mab E7 against $\beta$-tubulin was from the Developmental Studies Hybridoma Bank (University of Iowa). All fluorescent samples were mounted in Vectashield (Vector Laboratories).

Colocalization quantitation. Colocalization between Bchs and different GFP fusion markers in aCC and RP2 motor neuron cell bodies in embryonic CNS was calculated using ImageJ (rsb.info.nih.gov/ij) with the plugins: "Colocalization Test" and "Colocalization Threshold" by T. Collins (Wright Cell Imaging Facility, Toronto Western Research Institute, To- ronto, Ontario, Canada) and W. Rasband (Research Services Branch, National Institute of Mental Health, National Institutes of Health, Baltimore, MD), which were downloaded from the Wright Cell Imaging Facility, Toronto Western Research Institute (www.uhnresearch.ca/ facilities/wcif/imagej). Colocalization was expressed as three parameters: $\mathrm{R}$-coloc, the Pearson correlation coefficient, which varies between -1 and 1; the Manders coefficients tM1 for channel 1 (in this case the red channel, Bchs) and tM2 for channel 2 (the green channel, GFP), both of which are a fraction between 0 and 1 . Each of these values was calculated for pixels above a threshold level determined by the regression algorithm contained in the "Colocalization Threshold" macro, and applied to the intensity scatterplot of channel 1 versus channel 2 for each image. Each colocalization measurement was averaged from individual measurements of at least 72 images, in which individual GFP-positive cells had been selected as regions of interest (ROIs). For images in which Bchs background fluorescence was high, background was subtracted from the red channel using the "mean baseline" function in Leica SP2 confocal software. Data were compiled and graphed in Microsoft Excel.

Fly stocks. Blue cheese alleles used were as follows: $b c h s^{1}, b c h s^{e x 22}$ and $D f(2 L) c 17$, prl cn1/CyO (Finley et al., 2003), bchs ${ }^{58}$ (Khodosh et al., 2006), and Exel7024 (Berkeley Drosophila Genome Project). Transgenic fly stocks were RRa [eve-Gal4 (Fujioka et al., 2003)]; UAS-CD8-GFP (Lee and Luo, 1999), -spinster-GFP (Sweeney and Davis, 2002), -FYVE-GFP, -rab7-GFP (Wucherpfennig et al., 2003); -rab11-GFP (a gift from H. Chang, Biological Sciences, Purdue University, West Lafayette, IN); lysosome-associated membrane protein (LAMP)-GFP (Pulipparacharuvil et al., 2005); and -mito-GFP (a kind gift from A. Pilling and W. Saxton, Department of Molecular, Cell, and Developmental Biology, University of California, Santa Cruz, Santa Cruz, CA).

TUNEL staining and quantification. For Figure $7, b c h s^{58}, b c h s^{1}, b c h s^{e x 22}$, and wild type (WT) were taken at the same settings and magnification. The "auto-level" and median filtering functions were performed on the red channel for the images of $b c h s^{58}, b c h s^{1}, b c h s^{\text {ex22 }}$ in the figure.

TMR-red TUNEL stains (Roche) of Eve $>$ CD8-GFP-carrying thirdinstar larval brains for each mutant genotype were done according to manufacturer's instructions, side by side with wild-type brains. Briefly, single brains were dissected under HL3 saline and immediately transferred to ice. After 20 brains had been collected, they were fixed in $4 \%$ paraformaldehyde/PBS on ice for $30 \mathrm{~min}$, washed in $0.1 \% \mathrm{Na}$-citrate, $\mathrm{pH}$ $6 / 0.1 \%$ Triton X-100 several times, and left to permeabilize in this buffer on ice for $1 \mathrm{~h}$. They were then washed in PBTx $(1 \times \mathrm{PBS} / 0.1 \%$ Triton $\mathrm{X}-100)$, incubated in rabbit anti-GFP (Clontech) overnight at $4^{\circ} \mathrm{C}$, and washed again thoroughly in PBTx; at this point, several brains were removed from the wild-type batch for treatment with DNase $(3 \mathrm{U} / \mathrm{ml}$ in 50 $\mathrm{mm}$ Tris, $\mathrm{pH} 7.5 / 1 \mathrm{mg} / \mathrm{ml} \mathrm{BSA}$ ) as a positive control for TUNEL staining, for $10 \mathrm{~min}$ at $25^{\circ} \mathrm{C}$. DNase-treated brains were washed and placed together with remaining untreated wild-type brains for TUNEL staining. Brains were incubated $2 \mathrm{~h}$ at room temperature in Alexa 488-coupled anti-rabbit for detection of GFP, washed thoroughly in PBTx, rinsed in PBS twice, and then incubated in the TUNEL reagent for $60 \mathrm{~min}$ at $25^{\circ} \mathrm{C}$, rinsed in PBS, mounted, and imaged on a Leica SP2 confocal microscope with a $40 \times / 1.2$ numerical aperture (NA) oil-immersion lens using a pinhole setting of 1.23 airy units. Upper and lower limits of $0.5 \mu \mathrm{m}$ interval $Z$-stacks were set such that all GFP-expressing cells in the brain (aCC and RP2) were captured. Identical settings were used for WT and mutant. For quantification of WT versus $b c h s^{I} / D f(2) c 17$ TUNEL levels, single slices were analyzed on NIH ImageJ software, by measuring red (TUNEL) channel intensity value histograms from ROIs around individual GFP-positive nuclei. For measuring a given nucleus, a single image was taken from a $Z$-stack level in which that nucleus was clear in the corresponding green (GFP) image. Each nucleus was counted only once. Nuclei that had an average red intensity of $>20$ in WT or mutant were taken as positive for TUNEL. Since TUNEL intensities were much lower in the WT, red images from the WT only were autoleveled for intensity in Adobe Photoshop (Adobe Systems), before quantification. Although autoleveling would bias the data toward more TUNEL in the WT brains, WT levels were still quantitatively lower than mutants.

Live imaging. Flies for live imaging were cultured at $25^{\circ} \mathrm{C}$. Wandering third-instar larvae were anesthetized in Kwan Loong Medicated Oil 


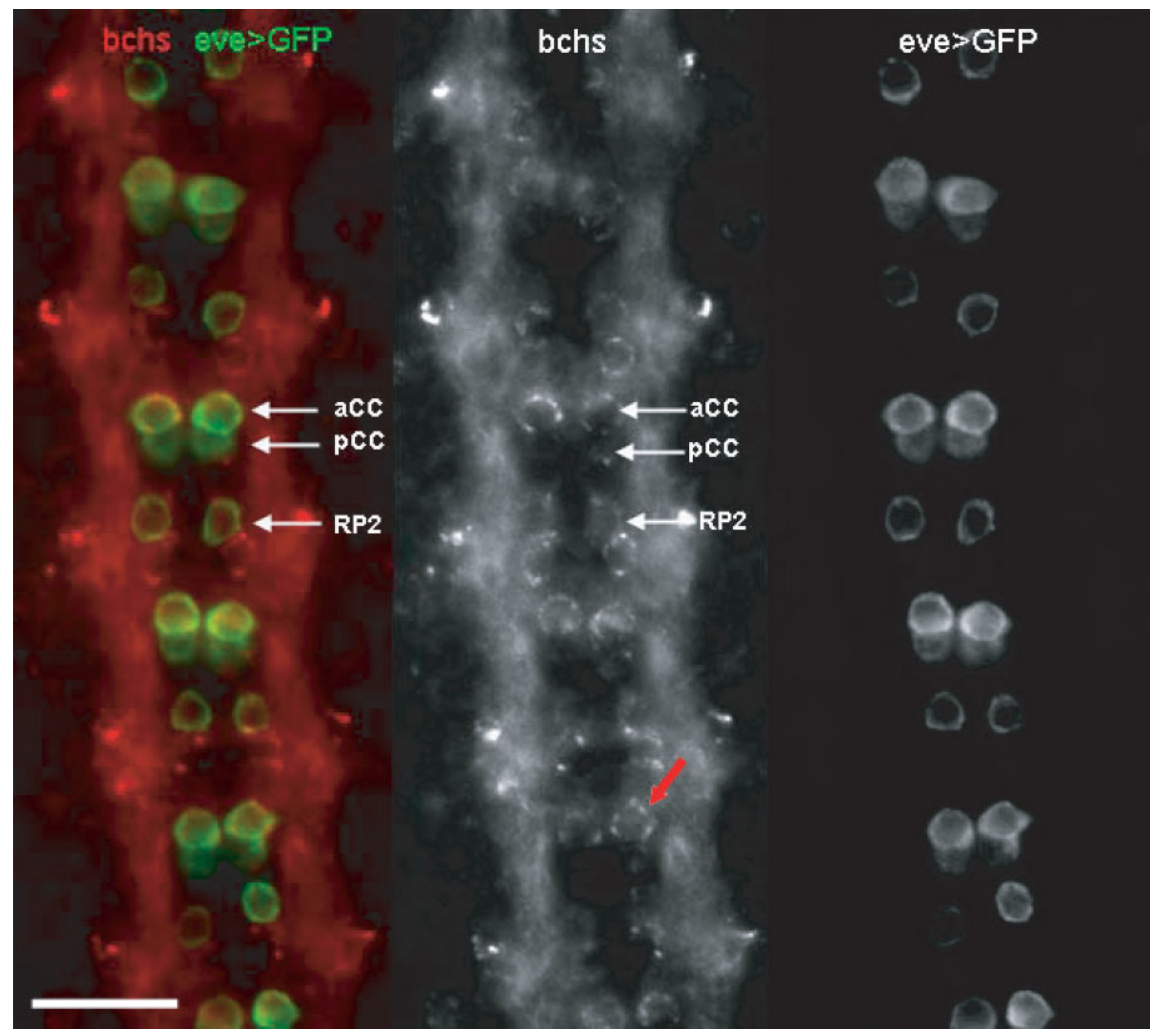

Figure 1. Bchs is expressed highly in a subset of motor neurons in embryonic CNS. Bchs (red) is seen in longitudinal axon tracts in the CNS and in a subset of motor neuron cell bodies in wide-field fluorescence images of a filleted stage 15/16 embryo. Note that punctate localization (red arrow) of Bchs is prominent in motor neuron cell bodies of aCC, RP2 (white arrows), and in an unidentified cell posterior to RP2. Images were acquired by wide-field fluorescence microscopy. Anterior is up. Scale bar, $20 \mu \mathrm{m}$. and Golgi, and at the synapse (Wang et al., 2000; Ward et al., 2000; Cornillon et al., 2002; Su et al., 2004; de Souza et al., 2007). Consistent with such a function, polyclonal antibody to the C-terminal $\sim 1000$ aa of the 3489 aa Drosophila Bchs protein (referred to as anti-Bchs), including the $\mathrm{BEACH}, \mathrm{WD} 40$, and FYVE domains, recognized intraneuronal vesicles in the embryonic CNS starting at approximately stage 12 , and continuing through embryogenesis (Fig. 1). The pattern of expression was similar to that of the bchs transcript by in situ RNA hybridization (data not shown) (Khodosh et al., 2006). Bchs protein is expressed in longitudinal connectives in the CNS, and in punctae in a subset of neuronal cell bodies that lie between the commissures (Fig. 1), but at low levels throughout the CNS, in muscles, and in other tissues. Two of these cell bodies were identifiable as the aCC and RP2 motor neurons by colocalization with an evenskipped Gal4 driver line combined with UAS-EGFP [referred to as eve $>$ EGFP (Fig. 1) (Broadus et al., 1995; Fujioka et al., 2003)]. The specificity of the antibody for Bchs was shown by the fact that genetic deficiencies that remove bchs showed extremely reduced protein expression, whereas overexpression of Bchs via EP(2)2299 (Rørth, 1996; Kraut et al., 2001) gave more protein recognized by (menthol, 25\%; methyl salicylate, 15\%; camphor, 10\%; eucalyptus oil, $10 \%$; lavender oil, $7 \%$; chloroform, $0.5 \%$ ) for $15 \mathrm{~min}$ and embedded in a drop of hemolymph-like saline [HL3 (Kuromi and Kidokoro, 1999)] plus $1 \%$ low-melting temperature agarose.

The aCC and RP2 axons of whole larvae were imaged on the Deltavision RT Restoration Microscopy System using $60 \times$ oil-immersion objectives, with immersion oil of 1.516 refractive index. Depending on the noise/signal level, images were acquired between 0.5 to $1 \mathrm{~s}$ per frame for up to $10 \mathrm{~min}$.

Image processing. Identified moving vesicles were tracked until they disappeared or could no longer be distinguished from other vesicles. Time-lapse images were aligned to correct for drift using a modified journal function in MetaMorph (Molecular Devices). Vesicles were tracked on the NIH ImageJ software using the Manual Tracker plug-in. To quantify number of moving and stationary vesicles, a threshold was performed using the "Count nuclei" journal before converting the resulting images to kymographs on the MetaMorph. For every 20 s segment, the number of moving and stationary vesicles were counted and expressed as percentage of total vesicles. Anterograde, retrograde, and stationary vesicles were also counted and expressed as a percentage of total moving vesicles. A vesicle was scored as anterograde or retrograde if it entered the $20 \mathrm{~s}$ segment and left it in the same direction; otherwise, it was scored as bidirectional. Because many of the vesicles in the mutants moved with frequent small changes in direction, the distance moved versus the actual displacement of each vesicle was measured. This was expressed as a fraction with values $>1$ indicating frequent changes in transport direction. Statistical analysis was performed with SigmaStat (SysStat Software), using one-way ANOVA testing or Student's $t$ test where indicated.

\section{Results}

Bchs protein is expressed on vesicles in a subset of neurons

The Bchs protein contains a BEACH domain, which occurs in a number of proteins involved in vesicle trafficking to lysosomes
anti-Bchs in embryonic fillet preparations (data not shown) and in Western blots (supplemental Fig. S1, available at www. jneurosci.org as supplemental material).

\section{Bchs colocalizes with endolysosomal markers}

To identify the vesicular compartments that contained the Bchs protein, transgenes for different GFP-fusion proteins that label various stages of the endolysosomal transport system were combined with the even-skipped GAL4 driver RRa [hereafter referred to as Eve $>$ marker (Fujioka et al., 2003)] in Bchs-positive motor neurons aCC and RP2. Embryos labeled in this way were filleted and stained with anti-Bchs, and the degree of colocalization with vesicle compartments was quantified. The Costes thresholding algorithm (Costes et al., 2004) (see Materials and Methods) was used to measure $R$, the Pearson correlation coefficient, and $M$, colocalizes with the other channel (Manders et al., 1993) in isolated cells in which both the GFP marker and Bchs punctae could be seen. Bchs showed the highest colocalization values with two late endolysosomal markers, LAMP-GFP (Pulipparacharuvil et al., 2005) and spinster-GFP (Sweeney and Davis, 2002) (Fig. $2 A, B)$. The early endosomal marker $2 \times$ FYVE-GFP, which bears two copies of the endosomal PI3P (phosphatidyl-insotol-3phosphate)-binding FYVE domain from Hrs (Wucherpfennig et al., 2003) displayed negative colocalization values for $R$, despite the fact that Bchs itself contains a FYVE domain (Fig. 2C). tM values were also lower for $2 \times$ FYVE-GFP, as well as for the late endosomal and multivesicular endosomal marker rab7-GFP (Entchev et al., 2000) (Fig. 2C,E). The recycling endosomal marker rab11-GFP (a gift from Henry Chang; communication to which reports on the percentage of intensity in each channel that 
Flybase FBrf0178891) showed a higher tM for colocalization with Bchs (green channel; tM2) but very little total colocalization, as judged by R-coloc (Fig. 2D,F). From the colocalization data, we conclude that Bchs is associated predominantly with a late endolysosomal compartment in motor neurons.

\section{Bchs mutation or overexpression leads to morphological abnormalities in motor neurons}

The motor nerves of $b c h s$-null mutant animals were examined for morphological defects in embryonic and larval stages. No obvious defects were detected in embryonic motor nerves, but by the third-instar larval stage, immunofluorescent staining for neuronal antigens FasII [a transmembrane protein that is expressed at the third-instar neuromuscular junction (NMJ)] and Futsch [a MAP1B ortholog, which associates with axonal and terminal microtubules (Hummel et al., 2000)] (Fig. $3 A-E$ ) showed abnormally thickened intersegmental nerves (ISNs) in bchs-null animals $\left[b c h s^{1}, b c h s^{E x e l 7024}\right.$ (Finley et al., 2003) (Drosophila Genome Research Consortium), or $b c h s^{58}$ in heterozygosity with the deficiency $D f(2 L) c 17$ (Khodosh et al., 2006)] (shown for bchs1/Df(2L)c17 and bchs58/Df(2L)c17 in Fig. 3). Average thickness at the thickest point below the ISN second branchpoint was measured and found to be $0.279 \pm 0.09$ for WT, $0.407 \pm$ 0.1 for $b c h s^{1} / D f(2 L) c 17$, and $0.373 \pm 0.1$ for $b c h s^{58} / D f(2 L) c 17$ (arbitrary units). The differences, although detectable, were not significant, because of a large variance within the population.

Using eve $>$ CD8-GFP as a membrane marker (Lee and Luo, 1999) expressed in only two motor neurons per hemisegment, we were able to examine the morphologies of individual axons in bchs larvae. Axonal membranes displayed irregular swellings and varicosities in bchs mutants, typical of degenerative phenotypes described in Alzheimer's and Wallerian degeneration models in mice (Kerschensteiner et al., 2005; Luo and O'Leary, 2005) (Fig. 3F-K).

Because disorganization and fragmentation of axonal microtubules are a hallmark of the Alzheimer's pathology (Fifre et al., 2006), we looked for differences in the morphology of labeled microtubules in bchs motor axons. Expression of Eve $>$ EGFP- $\alpha$ tubulin (Grieder et al., 2000) in aCC and RP2 (Fig. $4 A-F$ ) showed disruptions in axonal microtubule bundles and at termini. $\beta$-Tubulin immunostaining with MAbE7 (Fig. 4G,H) also showed disorganized microtubule bundles, particularly at the ISN branchpoint.

In addition to morphological changes in extant motor neu-

$\mathrm{F}_{0.5}$

0.4

0.3

0.2

0.1

0

$-0.1$
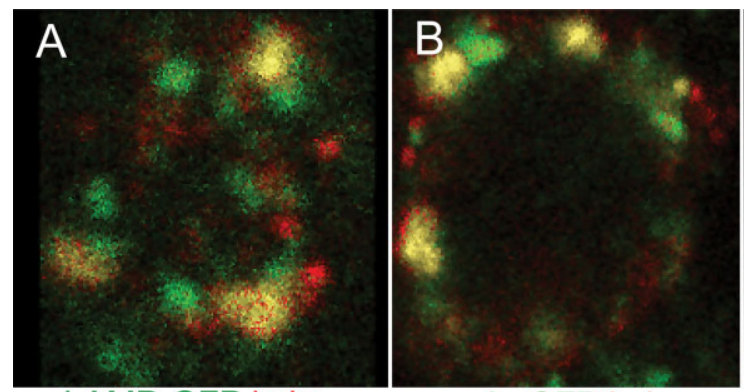

spin-GFP bchs

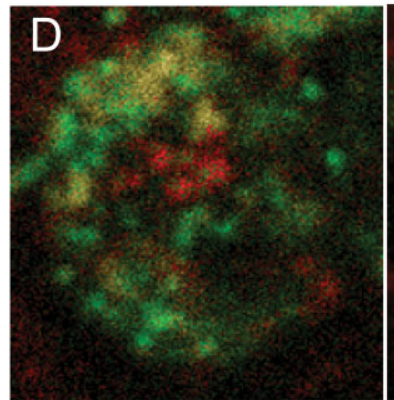

rab11-GFP bchs

\section{R-coloc}

Figure 2. Colocalization between Bchs and vesicular trafficking markers in embryonic aCC and RP2 motor neuron cell bodies. aCC and RP2 motor neurons were labeled with endolysosomal GFP fusion markers expressed via the Eve driver, and immunostained for endogenous Bchs in filleted stage 15/16 embryos. The Costes colocalization thresholding algorithm applied to the Pearson and Manders coefficients (part of the "Colocalization Threshold" ImageJ plugin by T. Collins and W. Rasband) was used to measure colocalization scores between Bchs and the endolysosomal markers LAMP-GFP $(\boldsymbol{A})(n=72)$ and spinster-GFP $(\boldsymbol{B})(n=$ 83); the endosomal sorting marker $2 \times$ FYVE-GFP $(\boldsymbol{C})(n=78)$; the recycling endosomal marker rab11-GFP $(\boldsymbol{D})(n=100)$; and the late endosome marker rab7-GFP $(E)(n=80)$ in single confocal images acquired with a $63 \times / 1.4$ NA objective lens, at $5 \times$ zoom. Overlaid red (Bchs) and green (GFP) channels show colocalized pixels in beige with intensity equivalent to $V$ /ch1 intensity * ${ }^{*}$ 2 2 intensity). $\boldsymbol{F}$, For each marker, thresholded values for the Pearson correlation coefficient (R-coloc), tM1 (the percentage of colocalized intensity in the red channel relative to total intensity in the red channel), and $\mathrm{M}$ 2 (the percentage of colocalized intensity in the green channel relative to total intensity in the green channel) are shown. Scores are plotted for pixels above an automatically determined threshold for both channels, according to the algorithm of Costes et al. (see Materials and Methods). Colocalization values for each parameter are consistently higher with late endolysosomal markers LAMP-GFP and spin-GFP than early endosomal or recycling markers FYVE-, rab7-, and rab11-GFP. Scale bar, $2 \mu \mathrm{m}$. Error bars show SEM. rons labeled with pan-neuronal markers and CD8-GFP, variable thinning, fading, or disappearance of the RP2 and also aCC labeled with CD8-GFP was observed (a range is shown in Fig. $5 A-F)$. This occurred in $b c h s^{58}$-null homozygotes, bchs nulls or hypomorphs over deficiency, as well as in animals overexpressing Bchs in RP2 and aCC (Fig. 5G). To determine whether apparent loss of the aCC and RP2 processes in the 

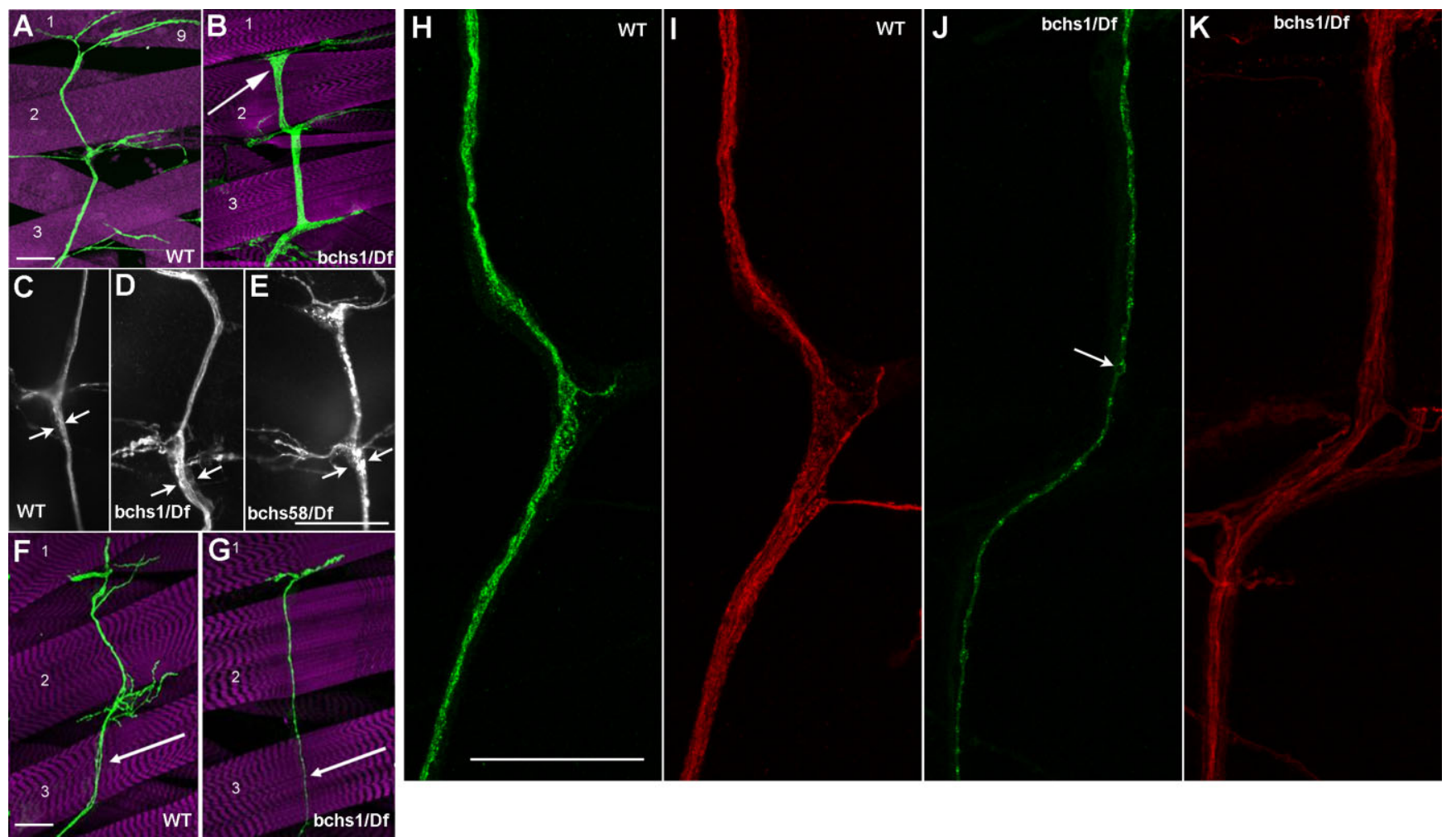

Figure 3. Abnormalities in ISN nerve bundles of $b$ chs-null larvae. $\boldsymbol{A}, \boldsymbol{B}$, WT (left) versus $b c h$ (right) ISN motor nerves terminating onto muscles $1,2,3$, and 9. Third-instar larval fillets were fixed and immunostained with anti-Futsch and Fasll (green) and rhodamine-phalloidin for muscles (purple), to show all fibers of the ISN. $\boldsymbol{B}$, In $b c h s^{1} / D f(2 L) c 17$ ISN swelling of the nerve fiber is evident (arrow) in this projected confocal image. $\boldsymbol{C}-\boldsymbol{E}$, Wide-field fluorescence images of Futsch/Fasll-labeled ISN nerves in WT (C), bchs ${ }^{1} / D f(2 L) c 17(\boldsymbol{D})$, and $b c h s 58 / D f(2 L) c 17(\boldsymbol{E})$, showing thickenings of the nerve bundle (arrows). F, G, WT (left) and $b c h s^{1} / D f(2 L) c 17$ (right) third-instar larval fillets expressing eve $>$ CD8-GFP only in aCC and RP2 motor neurons reveal apparent loss and thinning of individual fibers contributing to ISN nerve (arrow). Fillets were immunostained with anti-GFP and rhodamine-phalloidin (purple). $\boldsymbol{H}-\boldsymbol{K}$, Confocal images of eve $>$ CD8-GFP expressing aCC and RP2

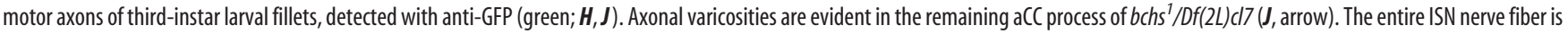
labeled with anti-Futsch and Fasll (red) $(\boldsymbol{I}, \boldsymbol{K})$. Scale bar, $40 \mu \mathrm{m}$ (in all images).

periphery was attributable to loss of GFP expression, or actual loss of neurons, CD8-GFP expression in RP2 and aCC cell bodies was observed simultaneously with endogenous Eve protein expression in the brains of wild-type and mutant Eve $>$ CD8-GFP expressing third-instar larvae (Fig. 5H-J). This showed frequent loss of one or more of the three Eve-positive nuclei per hemisegment that are seen in wild-type brains. Loss of GFP and retention of Eve in nuclei was also observed, suggesting that the loss of GFP in aCC and RP2 motor neurons is attributable to a combination of motor neuron death, and GFP suppression or degradation. The general loss of GFP was not specific to the Eve promoter, however, because other drivers in a bchs mutant background also showed variably reduced GFP levels (A. Wright and K. Zinn, personal communication).

Abnormal synaptic morphologies are also evident in bchs loss of function and overexpression (Fig. $5 D, E$ ), but were not studied in additional detail.

Atrophy of the RP2 motor neuron termini is shown in greater detail in intermediate cases in Figure 6. In these termini, two other fibers are detected in the vicinity of muscle 9 , in addition to RP2. In some cases, ISN termini and RP2 innervation appeared normal (Fig. 6C,D). In others, mainly loss of GFP occurred, but more often both GFP and futsch/FasII were reduced or absent (Fig. $6 F, G$ ). Incomplete cases of atrophy often showed that a second small fiber [apparently a type II ending (Johansen et al., 1989)] often grew closely along the path of the withered RP2 fiber [probably a type Is fiber, which is closer in size to the type II (Johansen et al., 1989; Gramates and Budnik, 1999)] (Fig. 6E,F). A much thicker fiber (presumably type Ib) did not express Eve $>$ GFP and can therefore not derive from RP2 (Fig. $6 B, D$ ). This larger type Ib fiber appears to be the same motor neuron designated 9Ib by Hoang and Chiba (2001).

\section{Bchs mutants show increases in TUNEL reactivity}

Because the extent of neuronal death was unclear from loss of GFP expression and other morphological changes alone, we performed TUNEL staining on bchs mutant and Bchsoverexpressing versus wild-type third-instar larval brains. Increased TUNEL reactivity occurs on DNA fragmentation in cells undergoing apoptosis, and in late stages of other types of cell death (de Torres et al., 1997). Both strong bchs alleles showed more TUNEL reactivity than the wild type (Fig. $7 A-C$ ). This was quantified for $b c h s^{1} / D f(2) c 17$, in which GFP-positive aCC/RP2 neurons were also scored for the presence or absence of TUNEL in their nuclei (51.6\% were TUNEL positive vs 3.5\% for the WT) (Fig. 7G). A hypomorphic allele, bchs ${ }^{\text {ex22 }}$ (Finley et al., 2003), and overexpression of Bchs from EP2299 (Rørth, 1996) both showed less TUNEL staining than $b c h s^{1}$ or $b c h s^{58}$, consistent with their lower frequency of total GFP loss in RP2 ( $\sim 15$ and $\sim 25 \%)$ (Fig. $7 D, E)$. The sizes of the cell bodies of motor neurons labeled with GFP in the bchs brains also appeared to be smaller than those of wild-type brains (Fig. 7, compare $I, H$ ), consistent with the documented shrinkage of cells under degenerative conditions (Delshad and Al-Tiraihi, 2001). 

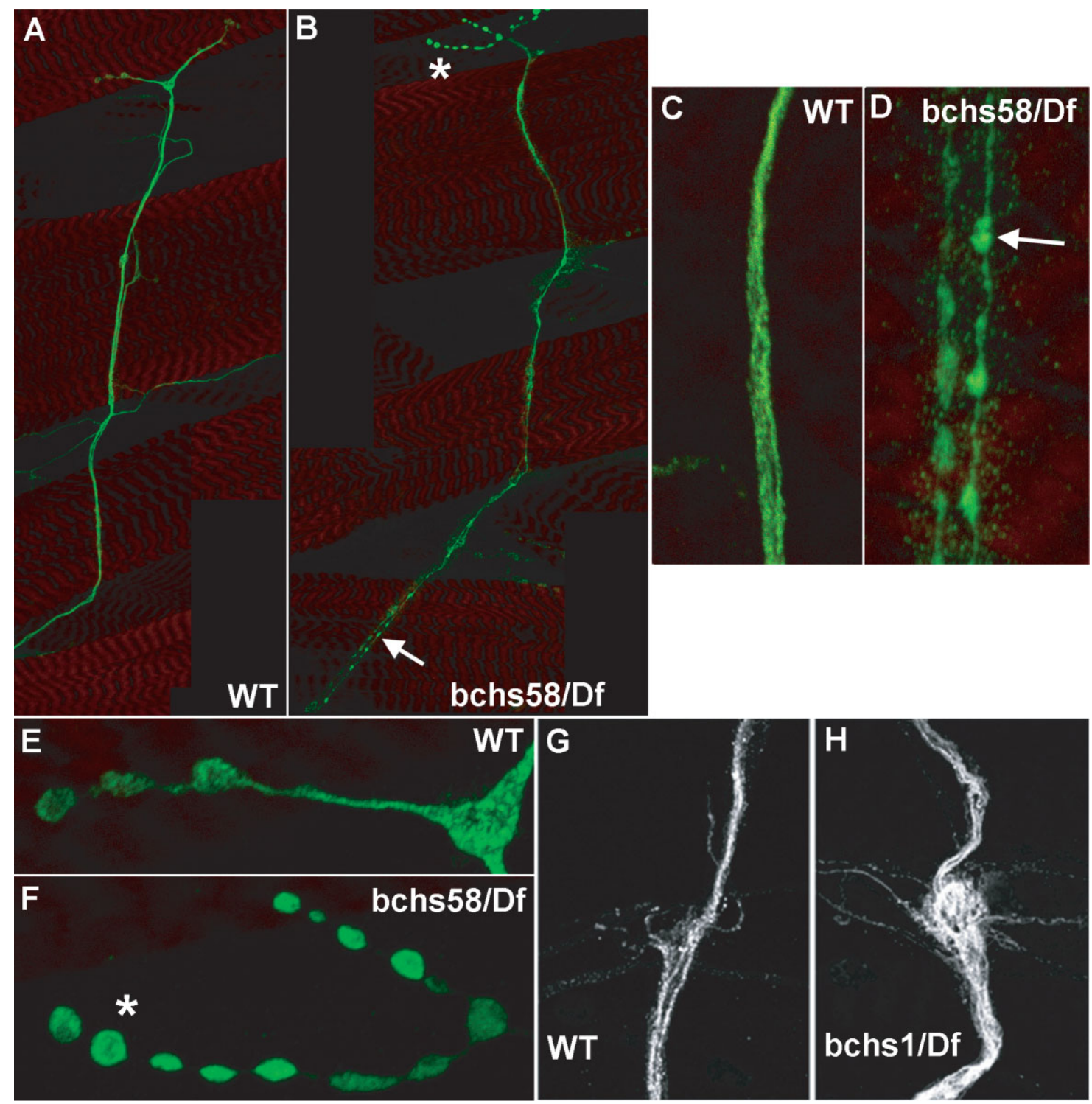

Figure 4. Microtubule bundles show abnormalities in bchs motor axons. $\boldsymbol{A}, \boldsymbol{B}$, Third-instar WT $(\boldsymbol{A})$ and $b c h 5^{58} / D f(2 L) c 17(\boldsymbol{B})$ third-instar larval fillets expressing eve $>G F P$ - $\alpha$-tubulin in aCC and RP2 motor neurons and immunostained with anti-GFP. Discontinuity of GFP expression is seen in the bchs axon fibers (arrow) and termini (asterisk), enlarged in $\boldsymbol{C}-\boldsymbol{F}$. $\boldsymbol{G}, \boldsymbol{H}, \mathbf{W T}$ (left) and bchs ${ }^{1} / D f(2 L)$ CI (right) ISN nerves at the level of the second branchpoint, showing abnormal organization of microtubules labeled with MAbE7 against $\beta$-tubulin.

Bchs affects anterograde transport of a spinster-positive lysosomal compartment

As mentioned above, Bchs shows high levels of colocalization with the late endolysosomal marker spinster-GFP [spin-GFP (Sweeney and Davis, 2002)]. Although the human Bchs ortholog Alfy has not been associated with any neurodegenerative disorder, fly Spinster and its human ortholog both have suspected roles in triggering cell death by a mechanism that is related to autophagy (Nakano et al., 2001; Yanagisawa et al., 2003). For this reason, and because Alfy is reported to be associated with autophagosomes (Simonsen et al., 2004), we looked for changes in the behavior of spin-GFP in bchs mutant and overexpressing animals. Because neurodegeneration is often associated with deficits in axon transport (Goldstein, 2001; Chevalier-Larsen and Holzbaur, 2006), we also looked for possible changes in the transport of spin-GFP vesicles.

To investigate the effects of Bchs on vesicle mobility, individual RP2 or aCC motor axons expressing spin-GFP vesicles were imaged in whole, intact third-instar larvae (Fig. 8) and vesicle runs were represented in kymographs (Fig. 8A) (see Materials and Methods). Spin-GFP vesicles were scored for mobility (moving vs stationary) and direction (anterograde, retrograde, and bidirectional) (Fig. 8C,E). Spin-GFP vesicles moved in both retrograde and anterograde directions in the aCC and RP2 motor axons, with the majority (55\%) moving anterograde in wild-type animals (Fig. $8 A, C$; supplemental Movie S1, available at www.jneurosci.org as supplemental material). However, in the mutants, most of the vesicles that moved did so in the retrograde direction (Fig. $8 \mathrm{~A}$, left panels, $C$; supplemental Movies S2, S3, available at www.jneurosci.org as supplemental material).

The $b c h s^{58}$-null mutation reduced the fraction of mobile vesicles substantially, from 42 to only $22 \%$, but $b c h s^{1}$ reduced mobility only slightly (from 42 to $39 \%$ ) (Fig. 8 E). The reason for the difference in severity between the two alleles is not entirely clear, given that both $b c h s^{1}$ and $b c h s^{58}$ behave as phenotypic nulls with respect to motor neuron atrophy and are protein nulls in Westerns (Khodosh et al., 2006; this study). 

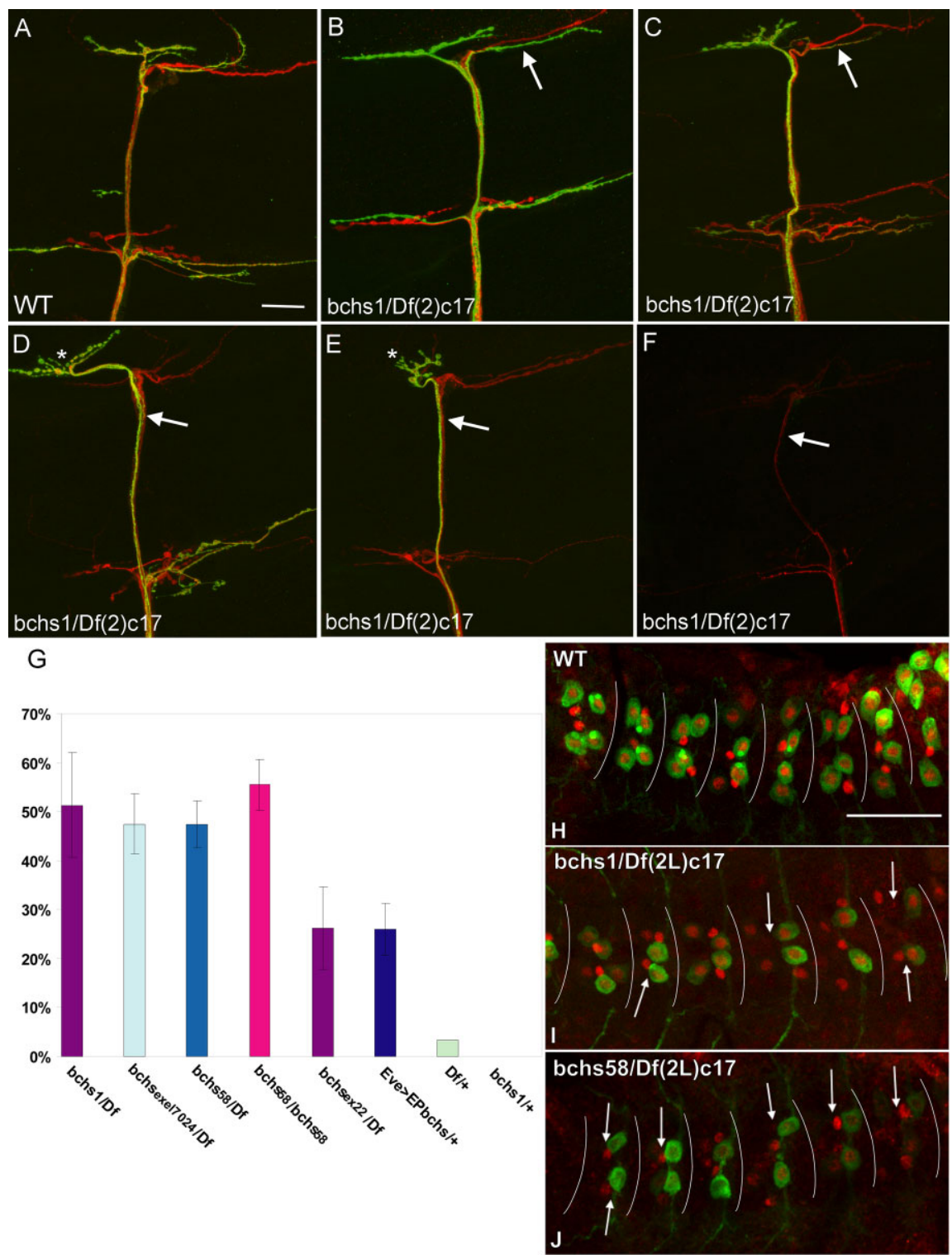

Figure 5. Loss of motor neurons in bchs-deficient ISN. $\boldsymbol{A}-\boldsymbol{F}$, Third-instar WT (A) and bchs 1/Df(2L) CI7 (B-F) larvae expressing eve $>$ CD8-GFP (green) in RP2 (arrows) and aCC and immunostained with anti-GFP. All motor neurons were also labeled with anti-Futsch/anti-Fasll (red). RP2 atrophy, as judged by loss of GFP and/or loss of Futsch/Fasll, has progressed to differing extents, from none evident $(\boldsymbol{B})$ to severe $(\boldsymbol{E}, \boldsymbol{F}$; arrows). In some cases, GFP levels were lowered, whereas Futsch/Fasll levels remained apparently normal. To see fibers, images should be viewed at high resolution (see supplemental material, available at www. jneurosci.org). G, Graph shows percentages of RP2 loss, scored as complete absence of detectable GFP, as in $\boldsymbol{E}$ and $\boldsymbol{F}$, in hemisegments of the genotypes shown. Error bars represent SD between three experiments. Asterisks point to morphological abnormalities in synaptic arbors. Scale bar, $40 \mu \mathrm{m}$ (in all images). $\boldsymbol{H}-\boldsymbol{J}$, Loss of GFP in motor neuron cell bodies of $b$ chs-deficient larval CNS is often accompanied by loss of Even-skipped. CNSs from eve $>$ CD8-GFP (green) third-instar larvae stained with antibody to endogenous eve (red), which localizes to cell nuclei. Wild-type $(\boldsymbol{H})$ third-instar CNS shows three eve-positive nuclei per hemisegment, two of which are GFP-positive aCC and RP2 (segments separated by drawn lines). I, bchs1/Df(2L)C17 CNS shows some missing eve nuclei (arrows point to segments in which eve nuclei are missing), in addition to loss of GFP. J, bchs58/Df(2L)c17 CNS shows similar, but more frequent loss of GFP and endogenous even-skipped.

Although $b c h s^{1}$ is a small deletion (for a molecular description of this and other deficiency alleles, see Finley et al., 2003), $b c h s^{58}$ has a stop codon at amino acid 1833 (Khodosh et al., 2006) and may make a truncated protein not recognized by the antibody. This could produce neomorphic phenotypes more severe than $b c h s^{1}$. For both $b c h s$ alleles, however, the primary defect was a reduction in the number of anterograde-moving vesicles, shifting net movement toward the retrograde direction (Fig. $8 C$ ). Overexpression via $E v e>E P(2) 2299$, which expresses the full-length Bchs protein (Kho- dosh et al., 2006), had the strongest effect on mobility, in which only $\sim 4 \%$ of vesicles moved (Fig. $8 \mathrm{E}$ ). Also, stationary vesicles appeared to accumulate in large clumps (supplemental Movie S4, available at www. jneurosci.org as supplemental material), although fluorescence imaging was of insufficient resolution to distinguish whether these were composed of enlarged vesicles or aggregated smaller vesicles. Bidirectional movement was never observed in Eve $>E P(2) 2299$ animals (Fig. 8C). Mobility was partially restored when Eve $>E P(2) 2299$ was expressed in a bchs-hemizygous $[D f(2) c 17 /+]$ background (Fig. $8 A$, right panels, $E$ ).

Movement in mutants was frequently interrupted with pauses and slight reverse movements (referred to as "jitter"). We quantified the degree of jitter among the different $b c h s$ mutants (Fig. $8 D$ ) by dividing the total distance moved by the actual displacement, for single vesicles. Vesicles that move more erratically have a jitter value $>1$. Jitter occurred in bchs loss-offunction animals, but was most apparent in $b c h s^{1}$ (Fig. 8D).

To determine whether transport velocity was affected in bchs mutants, individual vesicles were tracked over time, and velocities were calculated. Interestingly, bchs loss of function increased the average instantaneous velocities of the anterograde-moving vesicles (Fig. $9 A, B)$, whereas Bchs overexpression slightly decreased the velocities of the few vesicles that were moving. Movement of most tracked vesicles in $b c h s$ was also more saltatory and haphazard than in wild type. This is most apparent in the jagged trails from the kymographs (Fig. $8 A$; also see supplemental Movie S2, available at www.jneurosci.org as supplemental material). No differences were observed in the behavior of retrogrademoving vesicles in bchs (Fig. 9B).

To address the cargo specificity of Bchs effects on transport, we tracked the movements of mitochondria in aCC and RP2 motor neurons in a similar way, using eve $>$ mitoGFP in a wild-type background versus $b c h s^{58} / D f(2) c 17$, but found no effects on directionality of these organelles and only a slight reduction in mobility in the mutants $(p=$ 0.03 ) (supplemental Fig. $S 1 B-D$, available at www.jneurosci. org as supplemental material).

In summary, $b c h s$ loss of function strongly reduces the mobility of endolysosomal vesicles, and reverses the net direction of these vesicles from anterograde to retrograde, while increasing the transport velocity of the few vesicles that do move anterograde. $b c h s$ does not, however, affect the mobility or direction of mitochondria in the same motor axons, suggesting that the effect on transport is compartment specific. 

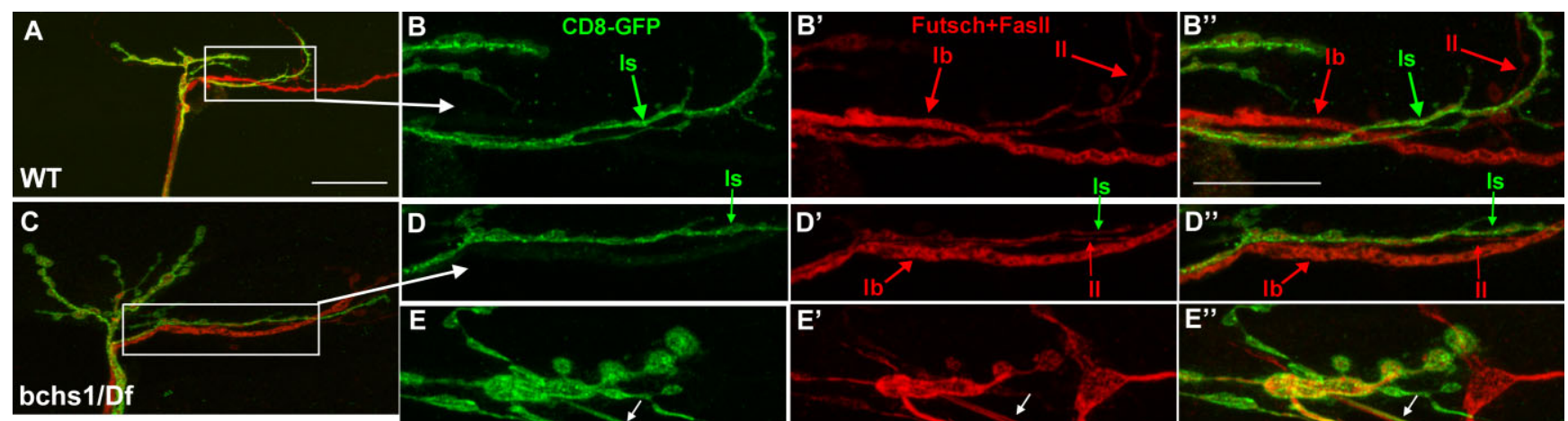

bchs1/D
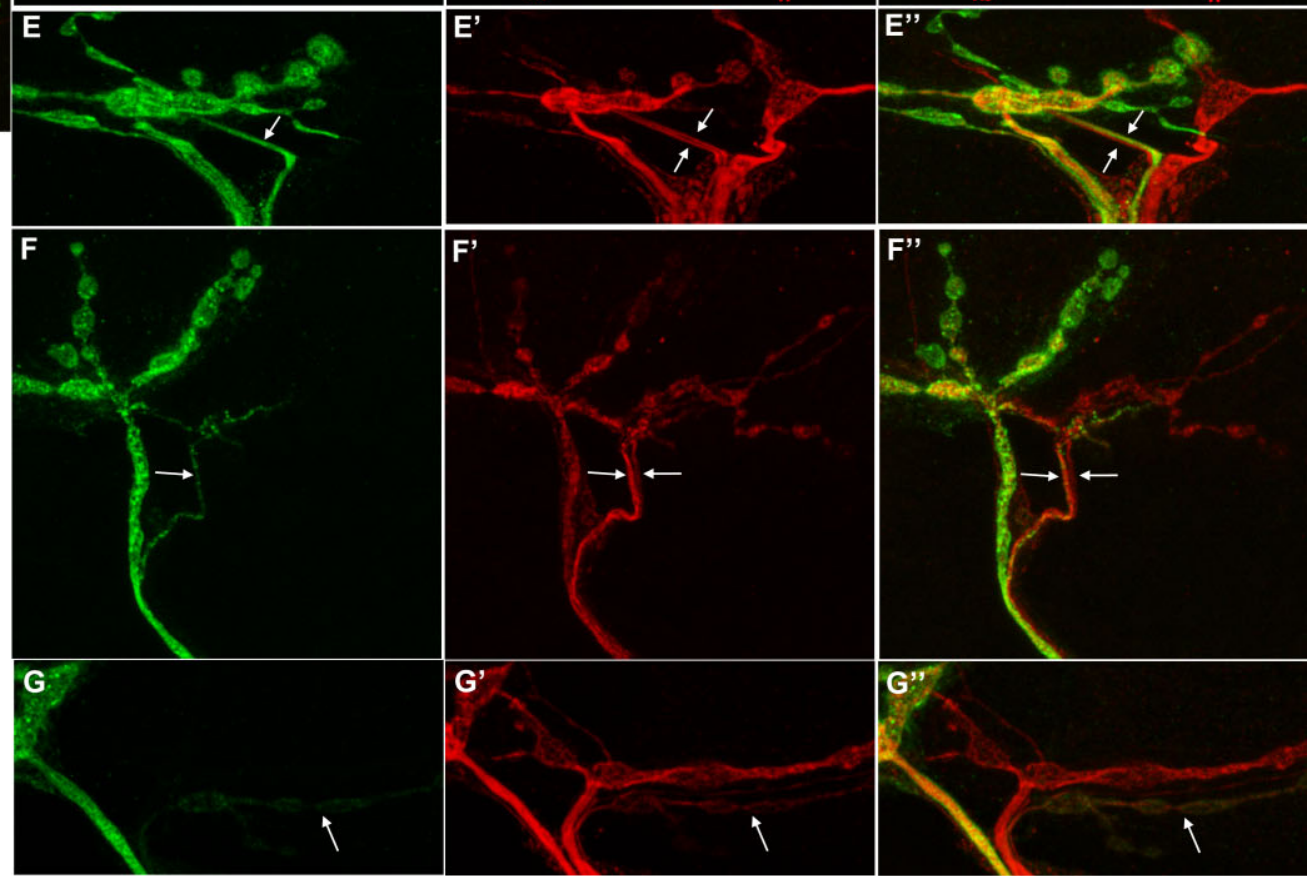

Figure 6. Enlarged details of motor neuron atrophy. $A$, Wild-type ISN terminal, showing aCC and RP2 motor neurons labeled by eve $>$ CD8-GFP (green), and other motor neurons stained for Futsch/Fasll (red). $\boldsymbol{B}-\boldsymbol{B}^{\prime \prime}$, Enlargement of $\boldsymbol{A}$. A non-GFP-labeled, Futsch/Fasll-positive small presumptive type ll terminal (red II, arrow) branches posteriorly and terminates close to the RP2 terminal (green Is, arrow). A larger presumptive type lb fiber (red lb, arrow) does not express GFP, showing that it does not originate from RP2.C, Abchs ${ }^{1} / D f(2 L) c 17$ terminal in which no motor neuron atrophy has occurred. $\mathbf{D}-\boldsymbol{D}^{\prime \prime}$, Enlargement of $\boldsymbol{C}$, showing a non-GFP-labeled presumptive type lb motor neuron (red lb; $\boldsymbol{D}^{\prime}$, arrow) that is morphologically similar to that in $\boldsymbol{B}^{\prime}$. As in $\boldsymbol{B}^{\prime \prime}, \mathrm{RP2}$ (green $\mathrm{ls} ; \boldsymbol{D}^{\prime \prime}$, arrow) is followed closely by a presumptive type II terminal (red $\| ; \boldsymbol{D}^{\prime}, \boldsymbol{D}^{\prime \prime}$, arrow). To see fibers, images should be viewed at high resolution (see supplemental material, available at www. jneurosci.org). $\boldsymbol{E}-\boldsymbol{E}^{\prime \prime}, A$ bchs $s^{1} / D f(2 L) c 17$ terminal showing RP2 (downward arrow) in close apposition to a second motor neuron fiber, presumably the type II (upward arrow). $\boldsymbol{F}-\boldsymbol{G}^{\prime \prime}, b c h s^{7} / D f(2 L) c 17$ termini in which diminution of both GFP and Futsch/Fasll has occurred (arrows), compared with levels in other fibers. Scale bars: $\boldsymbol{A}, 40 \mu \mathrm{m}$; enlarged images, $20 \mu \mathrm{m}$.

\section{Discussion}

A link between lysosomal defects and neurodegenerative disease, in particular Alzheimer's disease, has been recognized for over 10 years (Nixon et al., 2001). Degradation by the lysosome, but also other routes, is also required for the prevention of the toxic effects of aggregates that are involved in the polyglutamine disorders, Huntington's and Parkinson's disease (Rubinsztein, 2006). This association of degradative function with neurodegeneration became more acute recently with the discovery that loss of autophagic function, which overlaps with the lysosomal degradative pathway, leads to degeneration (Hara et al., 2006; Komatsu et al., 2006). A still unexplained phenomenon, however, is the relationship of lysosomes, and degradation in general, to axonal transport, which is also strongly affected in Alzheimer's, ALS, and other neurodegenerative diseases (Williamson and Cleveland, 1999; Kieran et al., 2005; Stokin and Goldstein, 2006; Lazarov et al., 2007) (for review, see Chevalier-Larsen and Holzbaur, 2006). In our current analysis of neurodegenerative defects in the motor nervous system of $b c h s$ mutants, we uncover a novel link between these two cellular functions, degradation and transport.

Bchs was previously known to be located on vesicles in motor neurons at the neuromuscular junction (Khodosh et al., 2006), and genetic interaction with rab11, a small GTPase associated with recycling endosomes, was shown. However, no evidence for a lysosomal function was demonstrated in that study, although Finley and colleagues (Simonsen et al., 2007) documented genetic suppression or enhancement of the rough eye phenotype induced by Bchs overexpression by mutations in lysosomal transport proteins. In light of these interactions, domain similarity of the BEACH family protein LysT, a lysosomal trafficking regulator mutated in Chediak-Higashi syndrome, suggested a possible role in vesicle trafficking to the lysosome, in addition to a vesicle recycling-related function. Our quantitative colocalization analysis of Bchs with different GFP fusion proteins that mark various steps in vesicular trafficking show the highest Pearson and Manders correlation coefficient values between the late endolysosomal proteins Spinster-GFP and LAMP-GFP (Sweeney and Davis, 2002; Pulipparacharuvil et al., 2005). Lower correlation values are seen with markers of other compartments such as rab7-GFP, FYVE-GFP, and rab11-GFP. From this, we conclude that, in embryonic motor neurons in which a degenerative mutant phenotype is evident, Bchs resides predominantly on an endolysosomal compartment.

In this study, we demonstrate the first direct evidence for a 

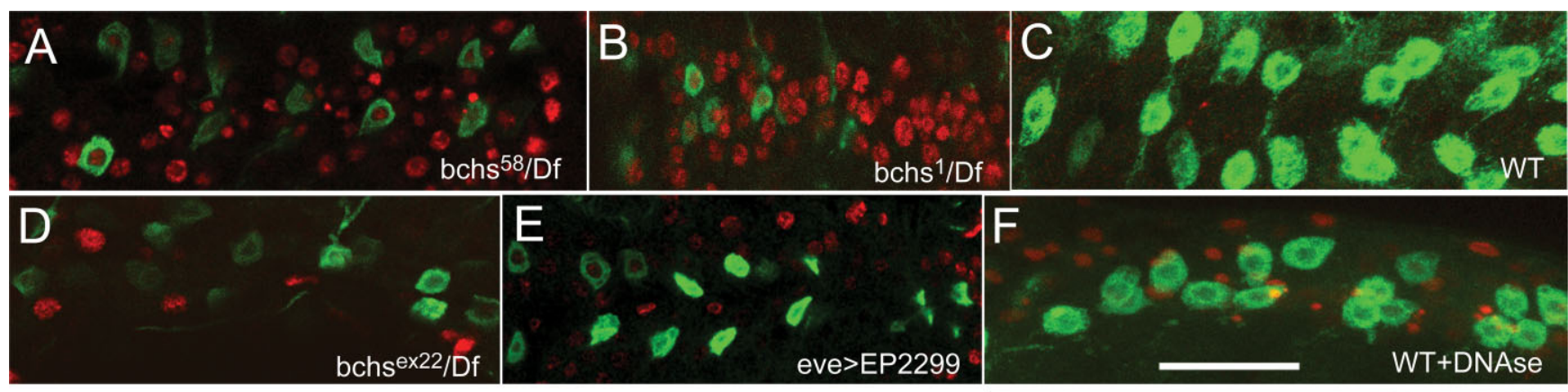

G
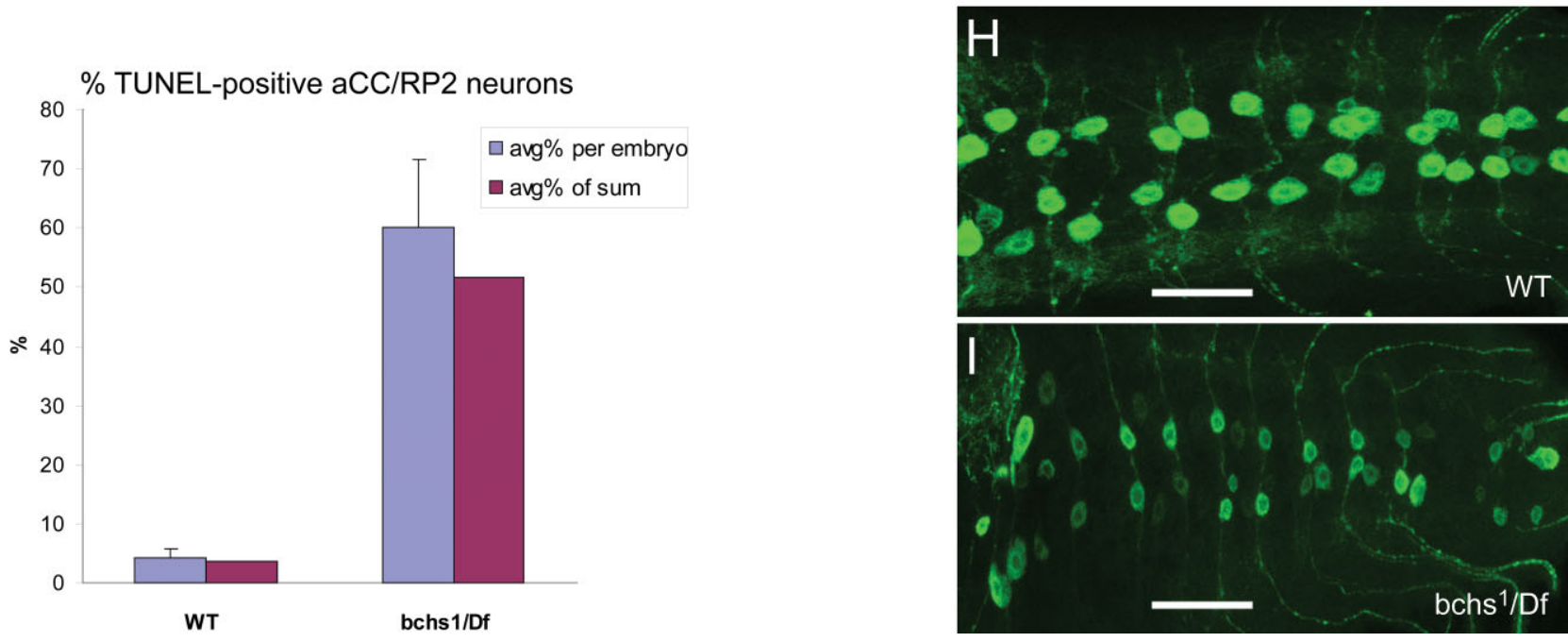

Figure 7. TUNEL reactivity is increased in $b c h s$ larval CNS. $A-F$, TUNEL (red) in CNS from $b c h s$ alleles $b c h s^{58}, b c h s^{1}, W T, b c h s^{\text {ex22 }}$, eve $>E P 2299$, and WT treated with DNase as a positive control for TUNEL reactivity. $b$ chs alleles are each in heterozygosity with $D f(2 L) c 17$. All brains express eve $>$ CD8-GFP, labeled with anti-GFP (green). Images in $b c h s$ mutant and overexpressing brains $(\boldsymbol{A}, \boldsymbol{B}, \boldsymbol{D}$, $E)$ are single confocal sections, and wild-type images $(\boldsymbol{C}, \boldsymbol{F})$ are projected stacks. The red (TUNEL) channel in all images was autoleveled in Adobe Photoshop, and median filtered, for presentation of images. G, Quantitation of CD8-GFP-positive aCC and RP2 nuclei that are also TUNEL positive in $b c h s^{1} / D f(2 L) c 17$ CNS. For WT, $n=311$ nuclei; for $b c h s^{1} / D f, n=128$ nuclei were scored. For quantitation of TUNEL signal, single confocal images of wild-type brain were autoleveled for the red (TUNEL) channel, but not for the bchs/Dfimages, such that any TUNEL signal in wild-type nuclei would be enhanced. Despite this, many fewer wild-type nuclei scored TUNEL positive (3.5\% average intensity $>20$ in ROI defined as a GFP-positive cell nucleus, vs $51.6 \%$ for $b c h s / D f)$. Each nucleus from a stack was counted only once, and all images were acquired at the same confocal settings and magnification. Error bars show SEM. H, I, Projections of wild-type and $b c h s^{1} / D f(2 L) c l 7$ brains $^{2}$ expressing eve $>$ CD8-GFP and labeled with anti-GFP, showing reduced neuronal size in the $b c h s^{1} / D f$ brain, which was acquired at the same magnification. Scale bars: $\boldsymbol{H}, \boldsymbol{I}, 40 \mu \mathrm{m}$.

lysosomal function of the Bchs protein, and document the degeneration of individual motor neurons in bchs mutant animals. Importantly, this is the first case in which a degenerative phenotype could be shown in identified neurons known to express the protein. $b c h s$ mutant CNS showed increases in TUNEL reactivity in general, and specifically, a loss among the RP2 and aCC motor neuron population in which Bchs is normally expressed at high levels. The loss of nuclei expressing Even-skipped, a marker for these neurons, also supports the conclusion that some of these motor neurons die. Surviving aCC and RP2 motor axons displayed varying extents of atrophy, axonal varicosities, and microtubule disorganization, typical for degenerating processes found in other neurodegenerative situations (Misgeld, 2005; Stokin et al., 2005; Fifre et al., 2006).

Moreover, in the same axons, severe defects in the transport of spinster-GFP-positive endolysosomes, but not GFP-tagged mitochondria, were observed in bchs mutants. This suggests not only that Bchs has a specific role in lysosomal trafficking but also provides a possible mechanism whereby transport defects may be associated with malfunction of a degradative compartment, resulting in degeneration.

Our data also reveal an interesting phenomenon that could be explained by regenerative or adventitious growth in the path of degenerating axonal processes. Inspection of the connectivity pattern of RP2/aCC (expressing CD8-GFP) against the total mo- tor neuron population (labeled with anti-Futsch/FasII) showed that a second motor neuron fiber of similar size often grew in close apposition to an atrophying fiber. This observation is consistent with the innervation of the dorsal muscles by multiple motor neurons (Schmid et al., 1999) and may suggest compensatory growth during degeneration. Selective vulnerability of certain motor neuron populations and adventitious growth after degeneration have also been reported in SOD mutant mouse models of ALS (Schaefer et al., 2005; Pun et al., 2006).

Association of lysosomal and autophagic dysfunction has been suggested in many studies, beginning with the observation that increased numbers of enlarged lysosomal and late endosomal compartments occurred in affected neurons in Alzheimer's brains (Cataldo et al., 1996). Subsequently, it was found that autophagosomes or multivesicular bodies, both of which fuse with lysosomes, are a site of $\mathrm{A} \beta$ production and accumulation (Takahashi et al., 2002; Yu et al., 2005). Together, a large body of evidence suggests that the function of lysosomes is critical in neurodegenerative pathologies, and this is especially evident in Alzheimer's disease (Nixon and Cataldo, 2006).

$b c h s$ mutants, as described here, present compelling evidence for a link between lysosomal function and axonal transport in neurodegeneration because identified degenerative motor neurons in these animals show strongly perturbed transport of endolysosomal vesicles. In the absence of Bchs, total mobility of 

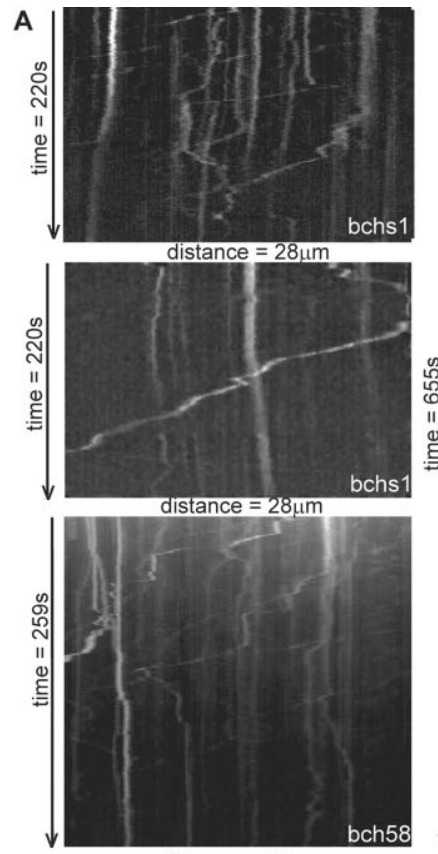

distance $=27 \mu \mathrm{m}$

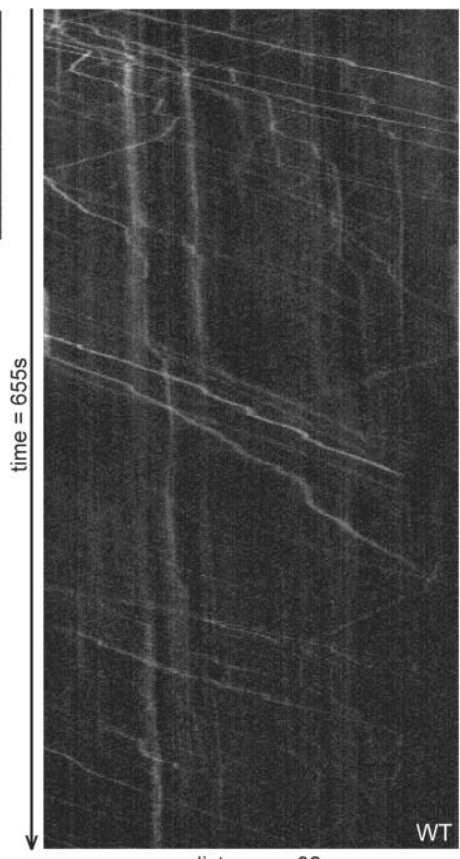

distance $=32 \mu \mathrm{m}$

C
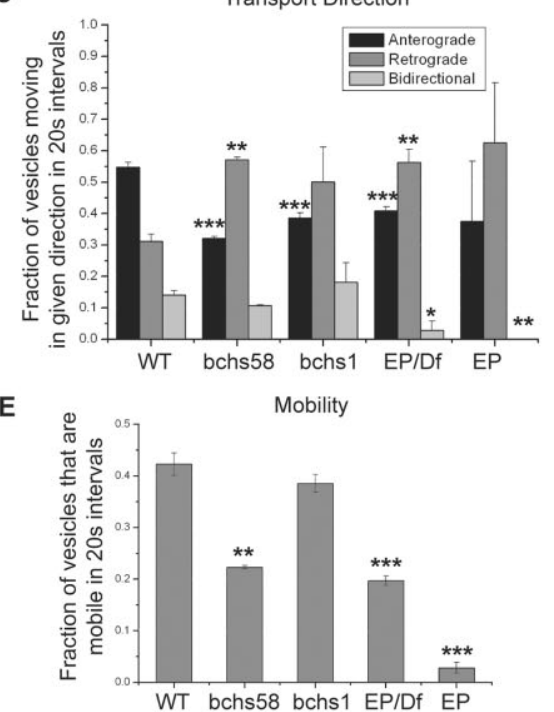

Figure 8. $\quad b c h$ s affects axonal transport of endolysosomes. $\boldsymbol{A}$, Kymographs of spinster-GFP-labeled endolysosomal vesicles undergoing anterograde and retrograde transport in RP2 or aCC motor axons in intact, anesthetized third-instar larvae. Representative kymographs are shown from time-lapse movies of various bchs loss-of-function and gain-of-function conditions. Time periods are shown (arrows) to the left of each frame, and distance encompassed by each movie is shown beneath each frame. Vesicles traveling in the anterograde direction are represented as slopes with negative gradient. Retrograde-directed vesicles are represented as slopes with positive gradient. Stationary vesicles are seen as vertical traces. bchs1, bchs ${ }^{1} / D f(2 L) c 17 ;$ bchs58, $b_{c h 5^{5}}$ /Df(2L)c17; EP, Eve > EP2299/+; EP/Df, Eve >EP2299/Df(2L)c17. Most vesicles in wild-type animals move in a relatively uninterrupted manner, whereas $b c h s^{1}$ and $b c h^{58}$ vesicles frequently pause and change direction. Transport in EP/ + axons is almost completely obliterated. EP/Df animals show partial rescue of the EP gain-of-function phenotype, with restoration of vesicle movement that approaches wild type. $\boldsymbol{B}$, Direction of transport in kymographs shown. $\boldsymbol{C}, \boldsymbol{b}$ chs reverses net transport direction. After thresholding the kymographs to select vesicles, numbers of anterograde, retrograde, and bidirectional moving vesicles were counted in 20 s segments $(n \geq 28$ ) of the kymograph and represented as a percentage of the total number of moving vesicles. The majority of vesicles in wild-type animals were anterograde directed (black bars). This trend was reversed in the $b c h s^{58}$ and $b c h s^{7}$ mutants, in which retrograde movement dominated (light gray bars). WT: ant, $55 \%$; ret, 31\%; bi, 14\%; bchs ${ }^{58}$ : ant, 32\%; ret, $57 \%$;

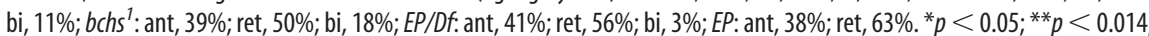
${ }^{* * *} p<0.0024$. D, bchs increases jitter of vesicle movement. The total distance traveled and the actual displacement of vesicles were measured and compared (distance/displacement $>$ or $<1$ ). Frequent changes in direction (jitter) give values of distance/ displacement $>1$. In $b c h s^{1}$ animals, $56 \%$ of vesicles had values $>1$, whereas in WT only $16 \%$ were $>1$. WT ( $n=96$ vesicles, 6 larvae); $b c h^{58}(49,6) ; b c h s^{1}(41,6), \operatorname{EP} / \operatorname{Df}(39,4) ; \operatorname{EP}(22,5) . \boldsymbol{E}$, bchs lowers overall endolysosomal mobility. Stationary and moving vesicles were counted in each 20 s interval of thresholded kymographs. Vesicle mobility is expressed as a percentage (number of moving vesicles/total vesicles $\times 100$ ). The majority of vesicles in the wild-type animals move anterograde. However, the trend is endolysosomal vesicles labeled by spinster-GFP is severely reduced. Moreover, anterograde transport is specifically suppressed, such that the net direction of transport shifts toward the cell body. When Bchs is overexpressed, transport in both directions is brought nearly to a complete stop.

That both Bchs loss of function and overexpression interfere with axonal transport in degenerative motor neurons strongly suggests a role for the Bchs protein in regulating transport, with anterograde transport being particularly affected. Furthermore, given the reported association of the human Bchs ortholog Alfy (Simonsen et al., 2004) with autophagosomes, and accumulation of ubiquitin-containing aggregates in bchs adult brain (Finley et al., 2003), we consider the possibility that the degradative function of late endolysosomal compartments at the NMJ is important in preventing atrophy of the neuron. In the absence of Bchs, anterograde transport and thus degradative activity at the NMJ may be inadequate, leading to motor neuron atrophy and death.

A parallel to this scenario has been described, in which autophagic clearance of aggregated proteins is crucial for preventing aggregate-induced neurodegeneration in fly and mouse models ( $\mathrm{Ra}-$ vikumar et al., 2002). These authors reported that the retrograde motor dynein is required for aggregate clearance by directing fusion of the autophagosome with the lysosome (Ravikumar et al., 2005). However, in the case of bchs, it appears to be anterograde transport that is involved in transporting a degradative (lysosomal) compartment to motor neuronal termini. It is not known whether some of the cargo transported by Bchs is also autophagosomal, and whether fusion is also affected.

Interestingly, the Bchs overexpression phenotype is similar to that seen on overexpression of the Drosophila neurodegenerative spastic paraplegia ortholog, Spicthyin (Wang et al., 2007). Spicthyin

\section{$\leftarrow$}

reversed in $b c h s$, in which retrograde movement dominates. WT $\left(n=35\right.$ segments counted); $b c h^{58}(n=29) ; b c h s^{1}(n=$ $28), \mathrm{EP} / \mathrm{Df}(n=33) ; \mathrm{EP}(n=31)$. Values of $p$ are as above, denoted by asterisks. Error bars show SEM. Genotypes are denoted as follows: bchs1, bchs ${ }^{1} / D f(2) c 17 ;$ bch58, $b c h^{58}$ / Df(2)c17; EP/Df, EP(2)2299/Df(2)c17; EP, EP(2)2299/+. All genotypes are in a background of two chromosomal copies of the eve driver and one copy of the UAS-spinster-GFP reporter. Direction of vesicles are denoted as follows: ant, anterograde; ret, retrograde; bi, bidirectional. 

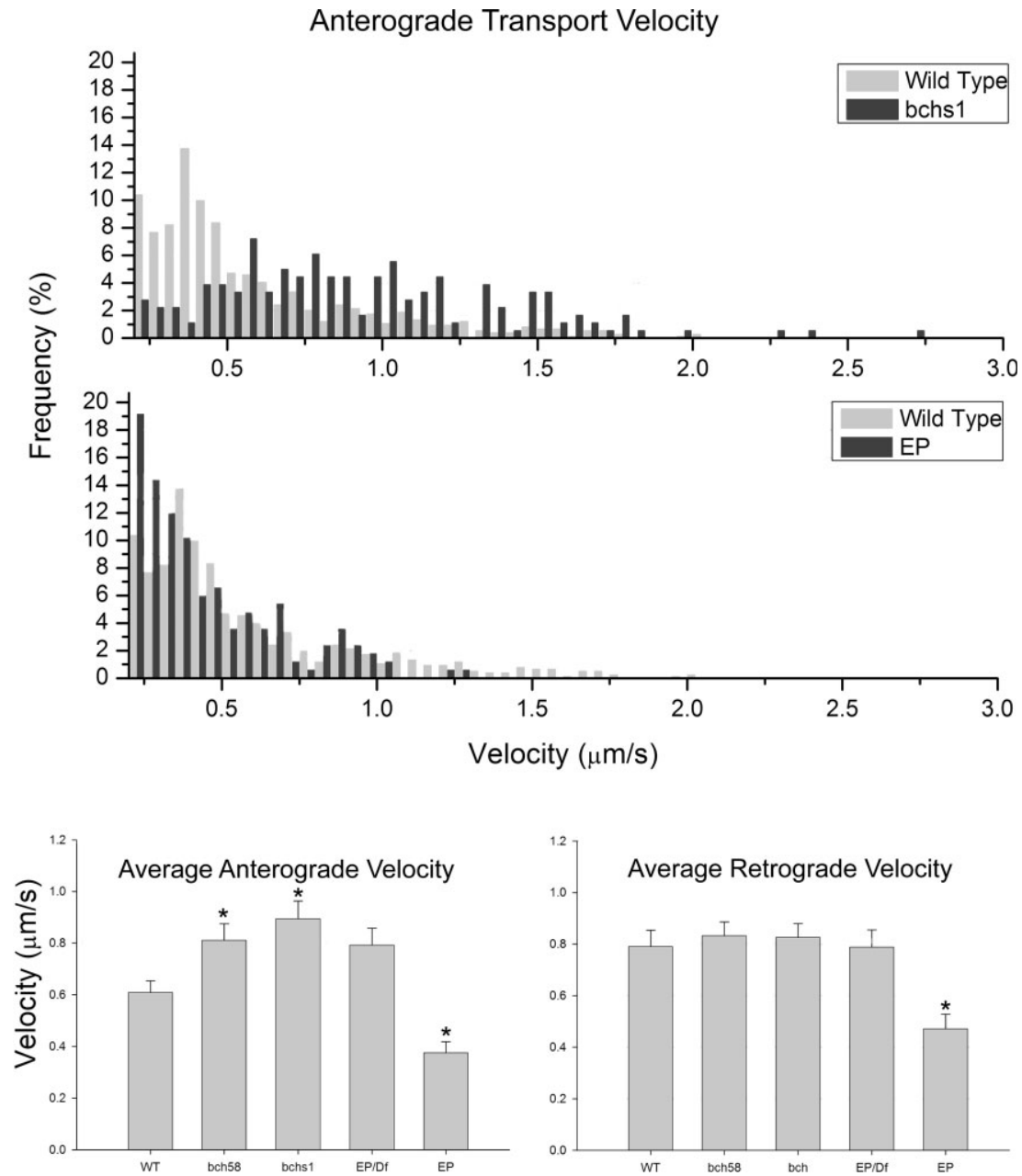

Figure 9. Effects of $b c h$ s on transport parameters. $\boldsymbol{A}$, Moving spin-GFP-positive vesicles in axons were tracked manually from time-lapse movies and the distance moved was used to calculate the instantaneous velocity of a given vesicle from one frame to the next. Instantaneous velocities were binned into groups in increments of $0.05 \mu \mathrm{m} / \mathrm{s}$ and presented as a frequency distribution histogram. Velocities were distributed over a range; however, there is a clear shift in bchs 1 toward higher velocities (rightward) and in eve $>$ EP2299/+ (EP) toward lower velocities (leftward). B (and Table 1), Instantaneous velocities measured over the length of a run for a given vesicle were used to calculate the average velocity of each tracked vesicle. Vesicles moving in the anterograde direction were more affected than those moving retrograde. Anterograde vesicles from $b c h 58$ and $b c h s 1$ animals move significantly faster, whereas in eve $>$ EP2299 animals, transport velocity in both directions was greatly reduced. Data are presented as means with SEM bars. ${ }^{*} p<0.05$, one-way ANOVA test.

Table 1. Average anterograde and retrograde velocities of spin-GFP vesicles in bchs mutant and overexpressing larvae

\begin{tabular}{|c|c|c|c|c|c|}
\hline & \multicolumn{5}{|l|}{ Genotype } \\
\hline & Wild type & $b c h^{58}$ & $b_{c h}{ }^{1}$ & $E P / D f$ & $E P$ \\
\hline \multicolumn{6}{|l|}{ Anterograde } \\
\hline No. of larvae & 6 & 6 & 6 & 4 & 5 \\
\hline Total particles tracked & 37 & 18 & 15 & 18 & 19 \\
\hline Points measured & 759 & 422 & 189 & 214 & 339 \\
\hline Mean & $0.609 \pm 0.0449$ & $\mathbf{0 . 8 1 1}^{*} \pm 0.0638$ & $0.894^{*} \pm 0.0686$ & $0.792 \pm 0.0658$ & $\mathbf{0 . 3 7 5 ^ { * } \pm 0 . 0 4 3 7}$ \\
\hline \multicolumn{6}{|l|}{ Retrograde } \\
\hline No. of larvae & 6 & 6 & 6 & 4 & 5 \\
\hline Total particles tracked & 21 & 30 & 28 & 16 & 8 \\
\hline Points measured & 262 & 692 & 508 & 172 & 127 \\
\hline Mean & $0.79 \pm 0.0643$ & $0.833 \pm 0.054$ & $0.826 \pm 0.054$ & $0.788 \pm 0.0678$ & $\mathbf{0 . 4 7 2}^{*} \pm 0.0569$ \\
\hline
\end{tabular}

Mean values are velocities $(\mu \mathrm{m} / \mathrm{s})$. Mean values in bold with asterisks are significantly different from wild type.

overexpression in motor neurons also halted axon transport and led to microtubule defects. However, no differences in axonal transport in spicthyin loss-of-function alleles were detected. In that study, the transport defects were attributed to an indirect effect on microtubules via TGF- $\beta$ signaling, which is modulated by Spicthyin. In contrast to Spicthyin mutants, bchs loss of function also affects axonal transport. For this reason, we suggest that Bchs may interact with a microtubule-associated motor or motors, acting as an adaptor between lysosomal vesicles and the transport machinery. Given that $b c h s$ does not appear to affect mitochondrial transport, we propose that Bchs interacts with a subset of cargoes including (but not necessarily limited to) endolysosomes, and is not generally required for transport along axons.

Although endolysosomal transport is less efficient in bchs motor axons, vesicles that do move actually have higher anterograde velocities, suggesting that anterograde transport may be regulated by retrograde tension. The loss of coordination between anterograde and retrograde motors in bchs may cause the erratic movement patterns of vesicles. Because mutations in both anterograde and retrograde motors can lead to defects in anterograde transport, and these motors interact with each other (Martin et al., 1999), the anterograde deficit seen in bchs could result from loss of regulation in either type of motor. In fact, Bchs could play a role similar to the Drosophila dynactin component, P150 ${ }^{\text {Glued }}$, which acts as a motor coordinating factor by preventing dynein and an anterograde motor from being active simultaneously (Gross, 2003).

An explanation for loss of anterograde transport in bchs may be offered by the recent findings of Holzbaur and colleagues (Dixit et al., 2008), who reported that accumulation of tau protein on microtubules affects anterograde and retrograde motors differently. On encountering a tau patch, kinesin falls off the microtubules, whereas dynein switches direction or slows down. Whether tau accumulates aberrantly in bchs mutants is as yet unknown.

Future work will be directed at determining which motor(s) Bchs may interact with, and whether Bchs participates in a vesicle transport "flotilla" such as that recently described (Horiuchi et al., 2007), whereby the JIP adaptor coordinates the assembly of a vesicle-bound receptor, kinesin and the MAPK (mitogen-activated protein kinase) cascade. Additionally, characterization of the identity of degradative compartments at the NMJ will lead to a better understanding of the relationship between lysosomal function and axon transport, and how failures in these processes lead to neurodegeneration. 


\section{References}

Bahr BA, Bendiske J (2002) The neuropathogenic contributions of lysosomal dysfunction. J Neurochem 83:481-489.

Broadus J, Skeath JB, Spana EP, Bossing T, Technau G, Doe CQ (1995) New neuroblast markers and the origin of the aCC/pCC neurons in the Drosophila central nervous system. Mech Dev 53:393-402.

Cataldo AM, Hamilton DJ, Barnett JL, Paskevich PA, Nixon RA (1996) Properties of the endosomal-lysosomal system in the human central nervous system: disturbances mark most neurons in populations at risk to degenerate in Alzheimer's disease. J Neurosci 16:186-199.

Chevalier-Larsen E, Holzbaur ELF (2006) Axonal transport and neurodegenerative disease. Biochim Biophys Acta 1762:1094-1108.

Cornillon S, Dubois A, Brückert F, Lefkir Y, Marchetti A, Benghezal M, De Lozanne A, Letourneur F, Cosson P (2002) Two members of the beige/ CHS (BEACH) family are involved at different stages in the organization of the endocytic pathway in Dictyostelium. J Cell Sci 115:737-744.

Costes SV, Daelemans D, Cho EH, Dobbin Z, Pavlakis G, Lockett S (2004) Automatic and quantitative measurement of protein-protein colocalization in live cells. Biophys J 86:3993-4003.

Cuervo AM, Stefanis L, Fredenburg R, Lansbury PT, Sulzer D (2004) Impaired degradation of mutant alpha-synuclein by chaperone-mediated autophagy. Science 305:1292-1295.

De Lozanne A (2003) The role of BEACH proteins in Dictyostelium. Traffic $4: 6-12$.

Delshad A, Al-Tiraihi T (2001) Ultrastructure of apoptotic oligodendrocytes in the spinal cord of adult rat with long-standing axotomised sciatic nerve. Folia Neuropathol 39:125-128.

de Souza N, Vallier LG, Fares H, Greenwald I (2007) SEL-2, the C. elegans neurobeachin/LRBA homolog, is a negative regulator of lin-12/Notch activity and affects endosomal traffic in polarized epithelial cells. Development 134:691-702.

de Torres C, Munell F, Ferrer I, Reventós J, Macaya A (1997) Identification of necrotic cell death by the TUNEL assay in the hypoxic-ischemic neonatal rat brain. Neurosci Lett 230:1-4.

Dixit R, Ross JL, Goldman YE, Holzbaur EL (2008) Differential regulation of dynein and kinesin motor proteins by tau. Science 319:1086-1089.

Entchev EV, Schwabedissen A, González-Gaitán M (2000) Gradient formation of the TGF-beta homolog Dpp. Cell 103:981-991.

Fifre A, Sponne I, Koziel V, Kriem B, Yen Potin FT, Bihain BE, Olivier JL, Oster T, Pillot T (2006) Microtubule-associated protein MAP1A, $\mathrm{MAP} 1 \mathrm{~B}$, and MAP2 proteolysis during soluble amyloid $\beta$-peptideinduced neuronal apoptosis: synergistic involvement of calpain and caspase-3. J Biol Chem 281:229-240.

Finley KD, Edeen PT, Cumming RC, Mardahl-Dumesnil MD, Taylor BJ, Rodriguez MH, Hwang CE, Benedetti M, McKeown M (2003) blue cheese mutations define a novel, conserved gene involved in progressive neural degeneration. J Neurosci 23:1254-1264.

Fujioka M, Lear BC, Landgraf M, Yusibova GL, Zhou J, Riley KM, Patel NH, Jaynes JB (2003) Even-skipped, acting as a repressor, regulates axonal projections in Drosophila. Development 130:5385-5400.

Goldstein LSB (2001) Kinesin molecular motors: transport pathways, receptors, and human disease. Proc Natl Acad Sci U S A 98:6999-7003.

Gramates LS, Budnik V (1999) Assembly and maturation of the Drosophila larval neuromuscular junction. Int Rev Neurobiol 43:93-117.

Grieder NC, de Cuevas M, Spradling AC (2000) The fusome organizes the microtubule network during oocyte differentiation in Drosophila. Development 127:4253-4264.

Gross SP (2003) Dynactin: coordinating motors with opposite inclinations. Curr Biol 13:R320-R322.

Gunawardena S, Goldstein LS (2004) Cargo-carrying motor vehicles on the neuronal highway: transport pathways and neurodegenerative disease. J Neurobiol 58:258-271.

Gunawardena S, Her LS, Brusch RG, Laymon RA, Niesman IR, GordeskyGold B, Sintasath L, Bonini NM, Goldstein LS (2003) Disruption of axonal transport by loss of huntingtin or expression of pathogenic polyQ proteins in Drosophila. Neuron 40:25-40.

Hafezparast M, Klocke R, Ruhrberg C, Marquardt A, Ahmad-Annuar A, Bowen S, Lalli G, Witherden AS, Hummerich H, Nicholson S, Morgan PJ, Oozageer R, Priestley JV, Averill S, King VR, Ball S, Peters J, Toda T, Yamamoto A, Hiraoka Y, et al. (2003) Mutations in dynein link motor neuron degeneration to defects in retrograde transport. Science 300:808-812.
Hara T, Nakamura K, Matsui M, Yamamoto A, Nakahara Y, SuzukiMigishima R, Yokoyama M, Mishima K, Saito I, Okano H, Mizushima N (2006) Suppression of basal autophagy in neural cells causes neurodegenerative disease in mice. Nature 441:885-889.

Hoang B, Chiba A (2001) Single-cell analysis of Drosophila larval neuromuscular synapses. Dev Biol 229:55-70.

Horiuchi D, Collins CA, Bhat P, Barkus RV, Diantonio A, Saxton WM (2007) Control of a kinesin-cargo linkage mechanism by JNK pathway kinases. Curr Biol 17:1313-1317.

Hummel T, Krukkert K, Roos J, Davis G, Klämbt C (2000) Drosophila Fut$\mathrm{sch} / 22 \mathrm{C} 10$ is a MAP1B-like protein required for dendritic and axonal development. Neuron 26:357-370.

Jablonka S, Wiese S, Sendtner M (2004) Axonal defects in mouse models of motoneuron disease. J Neurobiol 58:272-286.

Johansen J, Halpern ME, Johansen KM, Keshishian H (1989) Stereotypic morphology of glutamatergic synapses on identified muscle cells of Drosophila larvae. J Neurosci 9:710-725.

Kerschensteiner M, Schwab ME, Lichtman JW, Misgeld T (2005) In vivo imaging of axonal degeneration and regeneration in the injured spinal cord. Nat Med 11:572-577.

Khodosh R, Augsburger A, Schwarz TL, Garrity PA (2006) Bchs, a BEACH domain protein, antagonizes Rab11 in synapse morphogenesis and other developmental events. Development 133:4655-4665.

Kieran D, Hafezparast M, Bohnert S, Dick JR, Martin J, Schiavo G, Fisher EM, Greensmith L (2005) A mutation in dynein rescues axonal transport defects and extends the life span of ALS mice. J Cell Biol 169:561-567.

Komatsu M, Waguri S, Chiba T, Murata S, Iwata J, Tanida I, Ueno T, Koike M, Uchiyama Y, Kominami E, Tanaka K (2006) Loss of autophagy in the central nervous system causes neurodegeneration in mice. Nature 441:880-884.

Kraut R, Campos-Ortega JA (1996) inscuteable, a neural precursor gene of Drosophila, encodes a candidate for a cytoskeleton adaptor protein. Dev Biol 174:65-81.

Kraut R, Menon K, Zinn K (2001) A gain-of-function screen for genes controlling motor axon guidance and synaptogenesis in Drosophila. Curr Biol 11:417-430.

Kuromi H, Kidokoro Y (1999) The optically determined size of exo/endo cycling vesicle pool correlates with the quantal content at the neuromuscular junction of Drosophila larvae. J Neurosci 19:1557-1565.

LaMonte BH, Wallace KE, Holloway BA, Shelly SS, Ascaño J, Tokito M, Van Winkle T, Howland DS, Holzbaur EL (2002) Disruption of dynein/dynactin inhibits axonal transport in motor neurons causing late-onset progressive degeneration. Neuron 34:715-727.

Lazarov O, Morfini GA, Pigino G, Gadadhar A, Chen X, Robinson J, Ho H, Brady ST, Sisodia SS (2007) Impairments in fast axonal transport and motor neuron deficits in transgenic mice expressing familial Alzheimer's disease-linked mutant presenilin 1. J Neurosci 27:7011-7020.

Lee T, Luo L (1999) Mosaic analysis with a repressible cell marker for studies of gene function in neuronal morphogenesis. Neuron 22:451-461.

Luo L, O'Leary DD (2005) Axon retraction and degeneration in development and disease. Annu Rev Neurosci 28:127-156.

Manders EMM, Verbeek FJ, Aten JA (1993) Measurement of co-localization of objects in dual-color confocal images. J Microsc 169:375-382.

Martin M, Iyadurai SJ, Gassman A, Gindhart JG Jr, Hays TS, Saxton WM (1999) Cytoplasmic dynein, the dynactin complex, and kinesin are interdependent and essential for fast axonal transport. Mol Biol Cell 10:3717-3728.

Misgeld T (2005) Death of an axon: studying axon loss in development and disease. Histochem Cell Biol 124:189-196.

Nakano Y, Fujitani K, Kurihara J, Ragan J, Usui-Aoki K, Shimoda L, Lukacsovich T, Suzuki K, Sezaki M, Sano Y, Ueda R, Awano W, Kaneda M, Umeda M, Yamamoto D (2001) Mutations in the novel membrane protein spinster interfere with programmed cell death and cause neural degeneration in Drosophila melanogaster. Mol Cell Biol 21:3775-3788.

Nixon RA (2006) Autophagy in neurodegenerative disease: friend, foe or turncoat? Trends Neurosci 29:528-535.

Nixon RA, Cataldo AM (2006) Lysosomal system pathways: genes to neurodegeneration in Alzheimer's disease. J Alzheimers Dis 9:277-289.

Nixon RA, Mathews PM, Cataldo AM (2001) The neuronal endosomallysosomal system in Alzheimer's disease. J Alzheimers Dis 3:97-107.

Pulipparacharuvil S, Akbar MA, Ray S, Sevrioukov EA, Haberman AS, Rohrer 
J, Krämer H (2005) Drosophila Vps16A is required for trafficking to lysosomes and biogenesis of pigment granules. J Cell Sci 118:3663-3673.

Puls I, Jonnakuty C, LaMonte BH, Holzbaur EL, Tokito M, Mann E, Floeter MK, Bidus K, Drayna D, Oh SJ, Brown RH Jr, Ludlow CL, Fischbeck KH (2003) Mutant dynactin in motor neuron disease. Nat Genet 33:455-456.

Pun S, Santos AF, Saxena S, Xu L, Caroni P (2006) Selective vulnerability and pruning of phasic motoneuron axons in motoneuron disease alleviated by CNTF. Nat Neurosci 9:408-419.

Ravikumar B, Duden R, Rubinsztein DC (2002) Aggregate-prone proteins with polyglutamine and polyalanine expansions are degraded by autophagy. Hum Mol Genet 11:1107-1117.

Ravikumar B, Acevedo-Arozena A, Imarisio S, Berger Z, Vacher C, O'Kane CJ, Brown SD, Rubinsztein DC (2005) Dynein mutations impair autophagic clearance of aggregate-prone proteins. Nat Genet 37:771-776.

Rørth P (1996) A modular misexpression screen in Drosophila detecting tissue-specific phenotypes. Proc Natl Acad Sci U S A 93:12418-12422.

Rubinsztein DC (2006) The roles of intracellular protein-degradation pathways in neurodegeneration. Nature 443:780-786.

Salehi A, Delcroix JD, Belichenko PV, Zhan K, Wu C, Valletta JS, TakimotoKimura R, Kleschevnikov AM, Sambamurti K, Chung PP, Xia W, Villar A, Campbell WA, Kulnane LS, Nixon RA, Lamb BT, Epstein CJ, Stokin GB, Goldstein LS, Mobley WC (2006) Increased App expression in a mouse model of Down's syndrome disrupts NGF transport and causes cholinergic neuron degeneration. Neuron 51:29-42.

Schaefer AM, Sanes JR, Lichtman JW (2005) A compensatory subpopulation of motor neurons in a mouse model of amyotrophic lateral sclerosis. J Comp Neurol 490:209-219.

Schmid A, Chiba A, Doe CQ (1999) Clonal analysis of Drosophila embryonic neuroblasts: neural cell types, axon projections and muscle targets. Development 126:4653-4689.

Simonsen A, Birkeland HC, Gillooly DJ, Mizushima N, Kuma A, Yoshimori T, Slagsvold T, Brech A, Stenmark H (2004) Alfy, a novel FYVEdomain-containing protein associated with protein granules and autophagic membranes. J Cell Sci 117:4239-4251.

Simonsen A, Cumming RC, Lindmo K, Galaviz V, Cheng S, Rusten TE, Finley KD (2007) Genetic modifiers of the Drosophila blue cheese gene link defects in lysosomal transport with decreased life span and altered ubiquitinated-protein profiles. Genetics 176:1283-1297.

Small SA, Gandy S (2006) Sorting through the cell biology of Alzheimer's disease: intracellular pathways to pathogenesis. Neuron 52:15-31.

Stokin GB, Goldstein LSB (2006) Axonal transport and Alzheimer's disease. Annu Rev Biochem 75:607-627.

Stokin GB, Lillo C, Falzone TL, Brusch RG, Rockenstein E, Mount SL, Raman R, Davies P, Masliah E, Williams DS, Goldstein LS (2005) Axonopathy and transport deficits early in the pathogenesis of Alzheimer's disease. Science 307:1282-1288.

Su Y, Balice-Gordon RJ, Hess DM, Landsman DS, Minarcik J, Golden J, Hurwitz I, Liebhaber SA, Cooke NE (2004) Neurobeachin is essential for neuromuscular synaptic transmission. J Neurosci 24:3627-3636.

Sweeney ST, Davis GW (2002) Unrestricted synaptic growth in spinster-a late endosomal protein implicated in TGF-beta-mediated synaptic growth regulation. Neuron 36:403-416.

Szebenyi G, Morfini GA, Babcock A, Gould M, Selkoe K, Stenoien DL, Young M, Faber PW, MacDonald ME, McPhaul MJ, Brady ST (2003) Neuropathogenic forms of huntingtin and androgen receptor inhibit fast axonal transport. Neuron 40:41-52.

Takahashi RH, Milner TA, Li F, Nam EE, Edgar MA, Yamaguchi H, Beal MF, Xu H, Greengard P, Gouras GK (2002) Intraneuronal Alzheimer A $\beta 42$ accumulates in multivesicular bodies and is associated with synaptic pathology. Am J Pathol 161:1869-1879.

Wang N, Wu WI, De Lozanne A (2002) BEACH family of proteins: phylogenetic and functional analysis of six Dictyostelium BEACH proteins. J Cell Biochem 86:561-570.

Wang X, Herberg FW, Laue MM, Wullner C, Hu B, Petrasch-Parwez E, Kilimann MW (2000) Neurobeachin: a protein kinase A-anchoring, beige/Chediak-higashi protein homolog implicated in neuronal membrane traffic. J Neurosci 20:8551-8565.

Wang X, Shaw WR, Tsang HT, Reid E, O’Kane CJ (2007) Drosophila spichthyin inhibits BMP signaling and regulates synaptic growth and axonal microtubules. Nat Neurosci 10:177-185.

Ward DM, Griffiths GM, Stinchcombe JC, Kaplan J (2000) Analysis of the lysosomal storage disease Chediak-Higashi syndrome. Traffic 1:816-822.

Watts RJ, Hoopfer ED, Luo L (2003) Axon pruning during Drosophila metamorphosis: evidence for local degeneration and requirement of the ubiquitin-proteasome system. Neuron 38:871-885.

Williamson TL, Cleveland DW (1999) Slowing of axonal transport is a very early event in the toxicity of ALS-linked SOD1 mutants to motor neurons. Nat Neurosci 2:50-56.

Wucherpfennig T, Wilsch-Bräuninger M, González-Gaitán M (2003) Role of Drosophila Rab5 during endosomal trafficking at the synapse and evoked neurotransmitter release. J Cell Biol 161:609-624.

Yanagisawa H, Miyashita T, Nakano Y, Yamamoto D (2003) HSpin1, a transmembrane protein interacting with $\mathrm{Bcl}-2 / \mathrm{Bcl}-\mathrm{xL}$, induces a caspaseindependent autophagic cell death. Cell Death Differ 10:798-807.

Yu WH, Cuervo AM, Kumar A, Peterhoff CM, Schmidt SD, Lee JH, Mohan PS, Mercken M, Farmery MR, Tjernberg LO, Jiang Y, Duff K, Uchiyama Y, Näslund J, Mathews PM, Cataldo AM, Nixon RA (2005) Macroautophagy - a novel beta-amyloid peptide-generating pathway activated in Alzheimer's disease. J Cell Biol 171:87-98. 